\section{unesp}

UNIVERSIDADE ESTADUAL PAULISTA "JÚLIO DE MESQUITA FILHO"

Campus de São José do Rio Preto

\title{
Controle Exato Para a Equação de Onda. O Método HUM
}

\author{
José Henrique Rodrigues
}

Orientador: Prof. Dr. Waldemar Donizete Bastos

Dissertação apresentada ao Departamento de Matemática - IBILCE - UNESP, como parte dos requisitos para a obtenção do título de Mestre em Matemática

São José do Rio Preto

Fevereiro - 2010 
Rodrigues, José Henrique.

Controle exato para a equação de onda: o método Hum / José Henrique Rodrigues. - São José do Rio Preto : [s.n.], 2010.

$40 \mathrm{f} . ; 30 \mathrm{~cm}$.

Orientadora: Waldemar Donizete Bastos

Dissertação (mestrado) - Universidade Estadual Paulista, Instituto de Biociências, Letras e Ciências Exatas

1. Análise (Matemática). 2. Equações Diferenciais Parciais

3. Controle Exato.

I. Bastos, Waldemar Donizete. II. Universidade Estadual Paulista, Instituto de Biociências, Letras e Ciências Exatas. III. Título.

$$
\text { CDU - } 517
$$




\title{
JOSÉ HENRIQUE RODRIGUES
}

Controle Exato Para a Equação de Onda: O Método HUM

Dissertação apresentada para obtenção do título de Mestre em Matemática, junto ao Programa de PósGraduação em Matemática do Instituto de Biociências,

Letras e Ciências Exatas da Universidade Estadual Paulista "Júlio de Mesquita Filho", Campus de São José do Rio Preto.

\section{BANCA EXAMINADORA}

\author{
Waldemar Donizete Bastos \\ Professor Doutor \\ UNESP - São José do Rio Preto \\ Orientador
}

\section{Carlos Alberto Raposo da Cunha}

Professor Doutor

Universidade Federal de São João del Rei

\author{
Adalberto Spezamiglio \\ Professor Doutor \\ UNESP - São José do Rio Preto
}

São José do Rio Preto, 24 de fevereiro de 2010. 


\section{AGRADECIMENTOS}

Ao concluir este trabalho agradeço:

Primeiramente à Deus.

Ao meu orientador, Prof. Dr. Waldemar Donizete Bastos, pela paciência, pelas conversas, por me ajudar no conhecimento obtido sempre me indicando o caminho a ser seguido nos momentos de maior dificuldade e por depositar sua confiança em mim diante desse trabalho.

Aos meus pais Antônio Aparecido Rodrigues e Lusia de Andrade Rodrigues que, em meio a tantas dificuldades, forneceram seu apoio e suporte.

À banca examinadora.

À Ana, por estar sempre a meu lado.

À familia Michelan, minha segunda família, por me aceitarem como um legítimo membro desta família fornecendo todo seu apoio e suporte.

À todos que direta ou indiretamente contribuiram para a realização deste trabalho. 


\section{Resumo}

O objetivo deste trabalho é apresentar uma importante ferramenta no estudo da Teoria de Controle de Equações Diferenciais Parciais, o método HUM (Hilbert Uniqueness Method), introduzido por J. L. Lions em meados dos anos 80, ver [7] e [8].

Palavras-Chave: EDP, Controle, HUM. 


\section{Abstract}

The aim of this work is to present an important tool in the study of the Control Theory for Partial Differential Equations, cf. HUM method (Hilbert Uniqueness Method), introduced by J. L. Lions around the 80's, see [7] e [8].

Keywords: PDE, Control Theory, HUM 


\section{Sumário}

1 Conceitos Preliminares $\quad 10$

1.1 Os Espaços de Sobolev $\mathrm{H}^{m} \ldots \ldots \ldots$. . . . . . . . . . . . . . . 10

1.2 Funções a Valores Vetoriais . . . . . . . . . . . . . . . . . . . . . . . . . . . . . . . . . . . . . .

1.3 Outros Resultados . . . . . . . . . . . . . . . . . . 17

2 A Equação de Onda Linear $\quad 20$

2.1 Formulação do Problema . . . . . . . . . . . . . . . . . . . . . 20

2.2 Solução Ultrafraca . . . . . . . . . . . . . . . . . . . . . . . . . . . . . . . . . . . . . . . .

2.3 Resolução do Problema . . . . . . . . . . . . . . . . . . . . . . 34

$\begin{array}{ll}\text { Bibliografia } & 40\end{array}$ 


\section{Introdução}

Muitos fenômenos físicos podem ser modelados por problemas matemáticos envolvendo equações diferenciais parciais, em particular pelos problemas de evolução onde temos a presença de uma variável representado o tempo e uma variável representando as dimensões físicas do fenômeno em observação.

Uma vez modelado o fenômeno, buscaremos interferir no fenômeno por meio de um mecanismo, a ser chamado controle, a fim de obter uma solução do problema que, não apenas represente matematicamente o fenômeno, mas que possua condições inicial e final pré-determinadas. Mais precisamente, estudaremos o caso de controle na fronteira para fenômenos governados pela equação da onda. 


\section{Capítulo 1}

\section{Conceitos Preliminares}

Neste capítulo apresentaremos, de maneira sucinta, alguns dos conceitos básicos necessários ao que segue. Para maiores detalhes, recomendamos as referências aqui citadas e a bibliografia nestas contida.

\subsection{Os Espaços de Sobolev $\mathrm{H}^{m}$}

Nesta seção introduziremos alguns dos espaços de Sobolev e estabeleceremos algumas de suas propriedades básicas. Outros detalhes, bem como generalizações, podem ser obtidos em [1] ou ainda em [4].

Sejam $] a, b\left[\subset \mathbb{R}\right.$, um intervalo aberto e limitado, e $m \in \mathbb{N}^{*}$. Seja $u \in \mathrm{L}^{2}(a, b)$ uma função cujas derivadas distribucionais $D^{j} u, j=1, \ldots, m$ sejam também elementos de $\mathrm{L}^{2}(a, b)$. Para tais funções definimos o valor

$$
\|u\|_{m}=\left\{\sum_{j=0}^{m}\left\|D^{j} u\right\|^{2}\right\}^{\frac{1}{2}},
$$

onde $\|\cdot\|$ denota a norma usual de $\mathrm{L}^{2}(a, b)$.

Definimos o espaço de Sobolev $\mathrm{H}^{m}(a, b)$ como segue

$$
\mathrm{H}^{m}(a, b)=\left\{u \in \mathrm{L}^{2}(a, b) ; D^{j} u \in \mathrm{L}^{2}(a, b), \text { para } 1 \leqslant j \leqslant m\right\},
$$

Observamos que $\mathcal{C}^{m}(a, b) \subset \mathrm{H}^{m}(a, b)$. Além disso, a expressão (1.1.1) define uma norma em $\mathrm{H}^{m}(] a, b[)$.

Tem-se, portanto, o seguinte resultado.

Proposição 1.1.1. O espaço $H^{m}(a, b)$ munido da norma (1.1.1) é de Banach. 
Demonstração. Seja $\left(u_{\mu}\right)_{\mu \in \mathbb{N}^{*}}$ sequência de Cauchy em $\mathrm{H}^{m}(a, b)$. Pela definição da norma $\|\cdot\|_{m}$ segue que, para cada $j \in\{0,1, \ldots, m\},\left(D^{j} u_{\mu}\right)_{\mu \in \mathbb{N}^{*}}$ é sequência de Cauchy em $\mathrm{L}^{2}(a, b)$. Sendo este espaço completo, existem $v_{j} \in \mathrm{L}^{2}(a, b)$ tais que $D^{j} u_{\mu} \rightarrow v_{j}$ em $\mathrm{L}^{2}(a, b)$ e, conseqüentemente, em $\mathcal{D}^{\prime}(a, b)$. Em particular, para $j=0$ temos $u_{\mu} \rightarrow v_{0}$ em $\mathcal{D}^{\prime}(a, b)$. Logo, pela continuidade da derivação em $\mathcal{D}^{\prime}(a, b)$ segue que $D^{k} u_{\mu} \rightarrow D^{k} v_{0}$ em $\mathcal{D}^{\prime}(a, b)$ para qualquer $k \in\{0,1, \ldots, m\}$. Mas por unicidade do limite devemos ter $v_{j}=D^{j} v_{0}$ em $\mathcal{D}^{\prime}(a, b)$ para todo $j \in\{1, \ldots, m\}$. Assim, temos $v_{0} \in \mathrm{H}^{m}(a, b)$ e $u_{\mu} \rightarrow v_{0}$ neste espaço, o que conclui a demonstração.

Também não é difícil verificar que a aplicação

$$
(u, v)_{m}=\sum_{j=0}^{m}\left(D^{j} u, D^{j} v\right), \forall u, v \in \mathrm{H}^{m}(a, b) .
$$

é um produto interno em $\mathrm{H}^{m}(a, b)$ e que a norma (1.1.1) provém deste produto.

Assim, concluímos que $\mathrm{H}^{m}(a, b)$ munido da norma (1.1.1) e do produto interno (1.1.3) é um espaço de Hilbert.

Dentre os importantes resultados pertinentes aos espaços de Sobolev, destacamos o teorema de Meyers e Serin, publicado em 1964 e intitulado " $H=W$ ", por permitir formular, de maneira equivalente, o espaço $\mathrm{H}^{m}(a, b)$ como sendo o completamento de $\mathcal{C}^{m}(a, b)$ com relação a norma (1.1.1).

Outros resultados também de grande importância são as imersões de Sobolev. Entretanto, tais imersões são de difícil obtenção e podem ser encontradas na bibliografia citada antes. Porém, em se tratando de espaços de Sobolev definidos em intervalos limitados da reta, uma imersão se faz necessária e pode ser obtida sem muito esforço.

Primeiramente, lembramos que para dois espaços de Banach, digamos $\left(\mathrm{V},\|\cdot\|_{\mathrm{V}}\right)$ e $\left(\mathrm{U},\|\cdot\|_{\mathrm{U}}\right)$, sendo $\mathrm{V}$ subespaço de $\mathrm{U}$, dizemos que $\mathrm{V}$ está continuamente imerso em $\mathrm{H}$ quando existir $c>0$, constante, tal que $\|v\|_{\mathrm{H}} \leqslant c\|v\|_{\mathrm{V}}$, para todo $v \in \mathrm{V}$. Neste caso denotamos $\mathrm{V} \hookrightarrow \mathrm{H}$ para representar a imersão contínua. Podemos ver sem muita dificuldade que, pela definição de $\mathrm{H}^{m}(a, b)$ e pela norma (1.1.1) a este associada, temos $\mathrm{H}^{m}(a, b)$ continuamente imerso em $\mathrm{L}^{2}(a, b)$, e escrevemos $\mathrm{H}^{m}(a, b) \hookrightarrow \mathrm{L}^{2}(a, b)$. Além disso, são válidas ainda as imersões $\mathrm{H}^{j}(a, b) \hookrightarrow \mathrm{H}^{k}(a, b)$ para quais quer $j, k \in \mathbb{N}^{*} \operatorname{com} k<j$.

A seguir mostraremos que no caso de $m=1$ temos $\mathrm{H}^{1}(a, b)$ continuamente imerso em $\mathcal{C}([a, b]) . \operatorname{Em} \mathcal{C}([a, b])$ estamos considerando a norma da convergência uniforme, isto é, a norma dada por

$$
\|u\|_{\mathcal{C}([a, b])}=\max _{x \in[a, b]}|u(x)|, \forall u \in \mathcal{C}([a, b])
$$


Proposição 1.1.2. $H^{1}(a, b)$ está continuamente imerso em $\mathcal{C}([a, b])$.

Demonstração. Seja $u \in \mathrm{H}^{1}(a, b)$. Neste caso temos $u, D u \in \mathrm{L}^{2}(a, b)$. Fixando arbitrariamente $\left.x_{0} \in\right] a, b[$, definimos a função

$$
\left.v(x)=u(x)-\int_{x_{0}}^{x} D u(s) d s, \forall x \in\right] a, b[.
$$

É fácil ver que a derivada distribucional de $v$ é nula. Logo, segue que $v$ é constante em $\mathrm{L}^{2}(a, b)$, ou seja, existe $c \in \mathbb{R}$ tal que $v(x)=c$ em q.t.p. de $] a, b[$. Disto segue que

$$
\left.u(x)=c+\int_{x_{0}}^{x} D u(s) d s, \text { q.t.p. de }\right] a, b[.
$$

Isto prova que existe uma função contínua em $[a, b]$ que coincide com $u$ em q.t.p. do intervalo $[a, b]$. Neste sentido, $\mathrm{H}^{1}(a, b)$ está imerso em $\mathcal{C}([a, b])$.

Provaremos a seguir a continuidade da imersão. Seja $u \in \mathrm{H}^{1}(a, b)$. Pela primeira parte, podemos identificar $u$ com um elemento de $\mathcal{C}([a, b])$. Seja $x_{0} \in[a, b]$ tal que $\left|u\left(x_{0}\right)\right|=\min _{x \in[a, b]}|u(x)|$. Temos

$$
u(x)=u\left(x_{0}\right)+\int_{x_{0}}^{x} D u(s) d s, \forall x \in[a, b] .
$$

Observe que se $x_{0}=b$ então devemos mudar o sinal da integral. Logo, calculando o valor absoluto temos

$$
\begin{aligned}
|u(x)| & \leqslant\left|u\left(x_{0}\right)\right|+\int_{a}^{b}|D u(s)| d s=\frac{1}{b-a} \int_{a}^{b}\left|u\left(x_{0}\right)\right| d s+\int_{a}^{b}|D u(s)| d s \\
& \leqslant \frac{1}{b-a} \int_{a}^{b}|u(s)| d s+\int_{a}^{b}|D u(s)| d s, \forall x \in[a, b] .
\end{aligned}
$$

Utilizando em seguida a desigualdade de Schwarz temos

$$
|u(x)| \leqslant \frac{1}{(b-a)^{2}}\left\{\int_{a}^{b}|u(s)|^{2} d s\right\}^{\frac{1}{2}}+\frac{1}{b-a}\left\{\int_{a}^{b}|D u(s)|^{2} d s\right\}^{\frac{1}{2}}, \forall x \in[a, b],
$$

donde segue imediatamente que

$$
\max _{x \in[a, b]}|u(x)| \leqslant c\|u\|_{1}, \text { onde } c=\max \left\{\frac{1}{(b-a)^{2}}, \frac{1}{b-a}\right\},
$$

provando a continuidade da imersão.

Desta imersão podemos concluir o seguinte. 
Corolário 1.1.3. $H^{m}(a, b)$ está continuamente imerso em $\mathcal{C}^{m-1}([a, b])$.

Devido a imersão contínua de $\mathrm{H}^{1}(a, b)$ em $\mathcal{C}(a, b)$ é comum dizer, por abuso de linguagem, que os elementos $\mathrm{H}^{1}(a, b)$ são funções contínuas em $[a, b]$. Portanto, se $u \in \mathrm{H}^{1}(a, b)$ então podemos calcular $u(a)$ e $u(b)$, no sentido de que estes representam os valores da função contínua, a qual identifica-se com $u$, avaliadas nos respectivos pontos. A estes valores chamamos traço da função $u$. Em particular, destaca-se o subespaço das funções que possuem traço nulo, o qual será denotado por $\mathrm{H}_{0}^{1}(a, b)$, isto é, temos

$$
\mathrm{H}_{0}^{1}(a, b)=\left\{u \in \mathrm{H}^{1}(a, b) ; u(a)=u(b)=0\right\} .
$$

Entretanto, $\mathrm{H}_{0}^{1}(a, b)$ é comumente definido como sendo o completamento de $\mathcal{C}_{0}^{\infty}(a, b)$ com relação a norma $\|\cdot\|_{1}$. De maneira geral, definimos $\mathrm{H}_{0}^{m}(a, b)$ como sendo o completamento de $\mathcal{C}_{0}^{\infty}(a, b)$ com relação a norma $\|\cdot\|_{m}$.

Sabemos que o espaço $\mathrm{L}^{2}(a, b)$ é separável e reflexivo. Logo, estas propriedades se estendem aos espaços de Sobolev aqui definidos.

Proposição 1.1.4. Os espaços $H^{m}(a, b), m \in \mathbb{N}^{*}$, são reflexivos e separáveis.

Outros importantes espaços de Sobolev são os duais topológicos de $\mathrm{H}_{0}^{m}(a, b)$. Com efeito, denotaremos por $\mathrm{H}^{-m}(a, b)$ o dual topológico de $\mathrm{H}_{0}^{m}(a, b)$, ou seja,

$$
\mathrm{H}^{-m}(a, b)=\left\{f: \mathrm{H}_{0}^{m}(a, b) \rightarrow \mathbb{R} ; f \text { é linear e limitada }\right\}
$$

Devido a densidade de $\mathcal{C}_{0}^{\infty}(a, b)$ em $\mathrm{H}_{0}^{m}(a, b)$, o espaço $\mathrm{H}^{-m}(a, b)$ pode ser visto como subespaço de distribuições no intervalo $] a, b\left[\right.$, no sentido de que $\mathrm{H}^{-1}(a, b) \hookrightarrow \mathcal{D}^{\prime}(a, b)$. O resultado a seguir caracteriza o espaço $\mathrm{H}^{-m}(a, b)$.

Proposição 1.1.5. Seja $f \in \mathcal{D}^{\prime}(a, b)$. Então $f \in H^{-m}(a, b)$ se, e somente se, existirem funções $v_{0}, v_{1}, \cdots, v_{m} \in L^{2}(a, b)$ tais que $f=v_{0}+\sum_{j=1}^{m}(-1)^{j} D^{j} v_{j}$, ou seja,

$$
\langle f, u\rangle_{-1,1}=\left(v_{0}, u\right)+\sum_{j=1}^{m}(-1)^{j}\left(D^{j} v_{j}, D^{j} u\right), \forall u \in H_{0}^{m}(a, b)
$$

Além disso, temos

$$
\|f\|_{H^{-m}(a, b)}=\inf \left\{\left[\sum_{j=0}^{m}\left\|v_{j}\right\|^{2}\right]^{\frac{1}{2}} ; v_{0}, v_{1}, \ldots, v_{m} \in L^{2}(a, b) \text { satisfazem (1.1.13) }\right\}
$$


Temos portanto a seguinte cadeia de imersões

$$
\mathrm{H}_{0}^{m}(a, b) \hookrightarrow \mathrm{H}^{m}(a, b) \hookrightarrow \mathrm{L}^{2}(a, b) \hookrightarrow\left[\mathrm{H}^{m}(a, b)\right]^{\prime} \hookrightarrow \mathrm{H}^{-m}(a, b) \hookrightarrow \mathcal{D}^{\prime}(a, b)
$$

A seguir, apresentaremos a desigualdade de Poincaré-Friedrichs a fim de obtermos uma norma para $\mathrm{H}_{0}^{m}(a, b)$ que seja equivalente àquela definida por (1.1.1).

Lema 1.1.6 (Desigualdade de Poincaré-Friedrichs). Para toda função $u \in H_{0}^{1}(a, b)$ temos

$$
\|u\|^{2} \leqslant C\|D u\|^{2}
$$

onde $C>0$ é constante.

Demonstração. Seja $u \in \mathrm{H}_{0}^{1}(a, b)$ qualquer. Neste caso temos

$$
u(x)=\int_{a}^{x} D u(s) d s, \forall x \in[a, b] .
$$

Calculando em seguida o valor absoluto em (1.1.17) temos

$$
|u(x)| \leqslant \int_{a}^{b}|D u(s)| d s \leqslant \sqrt{b-a}\left\{\int_{a}^{b}|D u(s)|^{2} d s\right\}^{\frac{1}{2}}, \forall x \in[a, b] .
$$

Por fim, quadrando e integrando em $[a, b]$ obtemos

$$
\int_{a}^{b}|u(x)|^{2} d s \leqslant(b-a)^{2} \int_{a}^{b}|D u(s)|^{2} d s
$$

Portanto, basta tomar $C=(b-a)^{2}$ em (1.1.19) e o resultado segue.

Consideremos a seguinte aplicação

$$
\begin{aligned}
\|\cdot\|_{\nabla}: \mathrm{H}^{1}(a, b) & \rightarrow \mathbb{R} \\
u & \mapsto\|u\|_{\nabla}=\|D u\| .
\end{aligned}
$$

Não é difícil ver que (1.1.20) é norma em $\mathrm{H}^{1}(a, b)$.

Corolário 1.1.7. Em $H_{0}^{1}(a, b)$, as normas $\|\cdot\|_{\nabla} e\|\cdot\|_{1}$ são equivalentes.

Demonstração. Com efeito, se $u \in \mathrm{H}_{0}^{1}(a, b)$ então temos

$$
\|u\|_{1}^{2}=\|u\|^{2}+\|D u\|^{2} \leqslant C_{0}\|D u\|^{2} .
$$


onde $C_{0}=1+C$, com $C>0$ a constante obtida através da aplicação do lema de PoincaréFriedrichs. Por outro lado, a desigualdade $\|u\|_{1}^{2} \geqslant\|D u\|^{2}$ é válida para qualquer $u \in \mathrm{H}^{1}(a, b)$. Logo temos

$$
\|D u\|^{2} \leqslant\|u\|_{1}^{2} \leqslant C_{0}\|D u\|^{2}
$$

onde $C_{0}>0$ é constante. Estas desigualdades permitem concluir que em $\mathrm{H}_{0}^{1}(a, b)$ as normas $\|\cdot\|_{1}$ e $\|\cdot\|_{\nabla}$ são equivalentes.

De modo geral, definindo em $\mathrm{H}_{0}^{m}(a, b)$ a norma

$$
u \in \mathrm{H}_{0}^{m}(a, b) \mapsto\left\|D^{m} u\right\| \in \mathbb{R}
$$

podemos verificar facilmente que (1.1.1) e (1.1.23) são normas equivalentes em $\mathrm{H}_{0}^{m}(a, b)$.

Os resultados acima permitem concluir que o operador linear

$$
L: \mathrm{H}_{0}^{2 m}(a, b) \rightarrow \mathrm{H}^{-1}(a, b), \quad L=\sum_{j=1}^{m}(-1)^{j} D^{2 j}
$$

é um isomorfismo linear. Em particular, o operador de Laplace em dimensão 1, a dizer

$$
\begin{aligned}
-\Delta: \mathrm{H}_{0}^{1}(a, b) & \rightarrow \mathrm{H}^{-1}(a, b) \\
u & \mapsto-\Delta u=D^{2} u\left(=u_{x x}\right)
\end{aligned}
$$

é um isomorfismo linear.

\subsection{Funções a Valores Vetoriais}

Nesta seção faremos uma breve introdução às funções a valores vetoriais, isto é, funções definidas na reta e tomando valores em um espaço de Banach. As demonstrações dos resultados aqui apresentados podem ser encontrados em [6].

Sejam $X$ um espaço de Hilbert reflexivo e separável, com norma $\|\cdot\|_{\mathbf{X}}$ e produto interno $(\cdot, \cdot)_{X}$, e $] a, b\left[\right.$ um intervalo aberto e limitado de $\mathbb{R}$. Para $1 \leqslant p \leqslant \infty$, denotaremos por $\mathrm{L}^{p}(a, b ; \mathrm{X})$ o espaço das classes de funções $u:] a, b\left[\rightarrow \mathbf{X}\right.$ para as quais temos $\|u(\cdot)\|_{\mathbf{X}} \in \mathbf{L}^{p}(a, b)$. A estes espaços serão atribuídos as normas

$$
\|u\|_{\mathbf{L}^{p}(a, b ; \mathbf{X})}= \begin{cases}\left\{\int_{a}^{b}\|u(t)\|_{\mathbf{X}}^{p} d t\right\}^{\frac{1}{p}}, & \text { se } 1 \leqslant p<\infty \\ \operatorname{ess} \sup \|u(\cdot)\|_{\mathbf{X}}, & \text { se } p=\infty\end{cases}
$$


Proposição 1.2.1. Para $1 \leqslant p \leqslant \infty$ o espaço $L^{p}(a, b ; X)$, munido da norma (1.2.1), é Banach.

As propriedades de reflexibilidade e separabilidade pertinentes a $\mathbf{X}$ podem ser estendidas para o espaço $\mathrm{L}^{p}(a, b ; \mathbf{X})$ como descritas no próximo resultado.

Proposição 1.2.2. Sendo $X$ reflexivo e separável, segue que $L^{p}(a, b ; X)$ é separável, para $1 \leqslant$ $p<\infty$ e reflexivo, para $1<p<\infty$.

Seja $1 \leqslant p \leqslant \infty$. Chamaremos conjungado de $p$ ao número $p^{\prime}$, assim denotado, obtido da maneira seguinte

$$
p^{\prime}=\left\{\begin{array}{cl}
\infty, & \text { se } p=1 \\
\frac{p}{p-1}, & \text { se } 1<p<\infty \\
1, & \text { se } p=\infty
\end{array}\right.
$$

Formalmente, $p$ e $p^{\prime}$ satisfazem a equação $\frac{1}{p}+\frac{1}{p^{\prime}}=1$.

Denotando por $\mathbf{X}^{\prime}$ o dual topológico de $\mathbf{X}$, o próximo resultado fornece uma caracterização para o dual topológico de $\mathrm{L}^{p}(a, b ; \mathbf{X})$.

Proposição 1.2.3 (Phillips). Para $1 \leqslant p<\infty$, vale a identificação

$$
\left[L^{p}(a, b ; X)\right]^{\prime} \approx L^{p^{\prime}}\left(a, b ; X^{\prime}\right)
$$

onde $p^{\prime}$ é o conjugado de $p$. Para $p=\infty$ vale a inclusão

$$
\left[L^{\infty}(a, b ; X)\right]^{\prime} \subset L^{1}\left(a, b ; X^{\prime}\right)
$$

Proposição 1.2.4. Sejam $u \in L^{p}(a, b ; X)$ e $v \in L^{p^{\prime}}\left(a, b ; X^{\prime}\right)$, onde $1 \leqslant p \leqslant \infty$ e $p^{\prime}$ é conjugado de $p$. Então a função $t \in] a, b\left[\mapsto\langle v(t), u(t)\rangle_{-1,1} \in \mathbb{R}\right.$ é integrável à Lebesgue e ainda

$$
\int_{a}^{b}\langle v(t), u(t)\rangle_{-1,1} d t \leqslant\|u\|_{L^{p}(a, b ; X)}\|v\|_{L^{p^{\prime}}\left(a, b ; X^{\prime}\right)}
$$

onde $\langle\cdot, \cdot\rangle_{-1,1}$ denota a dualidade entre os espaços $X^{\prime}$ e $X$.

Seja agora $Y$ espaço de Hilbert separável e reflexivo, com norma $\|\cdot\|_{Y}$ e produto interno $(\cdot, \cdot)_{\mathrm{Y}}$. Por $\mathcal{L}(\mathrm{X}, \mathrm{Y})$ denotaremos o espaço das aplicações lineares e contínuas de $\mathrm{X}$ em $\mathrm{Y}$.

Proposição 1.2.5. Sejam $u \in L^{p}(a, b ; X)$ e $A \in \mathcal{L}(X, Y)$, com A fechado. Para $1 \leqslant p$ temos $A u \in L^{p}(a, b ; Y) e$

$$
\|A u\|_{L^{p}(a, b ; \gamma)} \leqslant\|A\|_{\mathcal{L}(X, Y)}\|u\|_{L^{p}(a, b ; X)}
$$


Em particular, para $p=1$ vale

$$
\int_{a}^{b} A u(t) d t=A \int_{a}^{b} u(t) d t
$$

Demonstração. Veja [5] página 3.

Observação 1.2.1. A integral acima é definida do mesmo modo que no caso $\mathbf{X}=\mathbb{R}$, isto é, como limite de somas de Riemann tomadas na norma $\|\cdot\|_{\mathbf{X}}$.

Corolário 1.2.6. Se $u \in L^{p}(a, b ; X)$ e $f \in X^{\prime}$, temos

$$
\left\langle f, \int_{a}^{b} u(t) d t\right\rangle_{-1,1}=\int_{a}^{b}\langle f, u(t)\rangle_{-1,1} d t
$$

onde $\langle\cdot, \cdot\rangle_{-1,1}$ representa a dualidade entre os espaço $X^{\prime}$ e $X$.

\subsection{Outros Resultados}

Nesta seção apresentaremos resultados técnicos necessários ao desenvolvimento do que segue.

Proposição 1.3.1. Sejam $m \in L^{1}(0, T)$, tal que $m(s) \geqslant 0$ em q.t.p. de $[0, T]$, e a $\geqslant 0$ constante real. Suponha que $g \in L^{\infty}(0, T)$ é tal que $g(t) \geqslant 0$ em $[0, T]$ e ainda

$$
\frac{1}{2} g(t)^{2} \leqslant 2 a^{2}+2 \int_{0}^{t} m(s) g(s) d s
$$

para todo $t \in] 0, T[$. Então

$$
g(t) \leqslant 2\left(a+\int_{0}^{t} m(s) d s\right), e m[0, T]
$$

Demonstração. Seja $\varepsilon>0$ arbitrário. Considere a seguinte função

$$
\psi_{\varepsilon}(t)=\sqrt{2}(a+\varepsilon)^{2}+2 \int_{0}^{t} m(s) g(s) d s, \text { para } t \in[0, T] .
$$

Observe que $\psi_{\varepsilon}(t)>0$ para todo $t \in[0, T]$. Ainda, derivando no sentido usual a expressão (1.3.3) obtemos

$$
\frac{d \psi_{\varepsilon}}{d t}(t)=2 m(t) g(t), \text { em q.t.p. de }[0, T]
$$


Por outro lado, é fácil ver que por (1.3.1) e (1.3.3) segue

$$
\frac{1}{2} g(t)^{2} \leqslant \psi_{\varepsilon}(t) \Longleftrightarrow g(t) \leqslant \sqrt{2 \psi_{\varepsilon}(t)} \Longleftrightarrow \frac{g(t)}{\sqrt{\psi_{\varepsilon}(t)}} \leqslant \sqrt{2},
$$

para todo $t \in[0, T]$. Assim, (1.3.4) e (1.3.5) implican

$$
\frac{d \sqrt{\psi_{\varepsilon}(t)}}{d t} \leqslant \sqrt{2} m(t), \text { em q.t.p. de }[0, T]
$$

Integrando (1.3.6) de 0 a $t \leqslant T$ obtemos

$$
\sqrt{\psi_{\varepsilon}(t)} \leqslant \sqrt{2}(a+\varepsilon)^{2}+\sqrt{2} \int_{0}^{t} m(s) d s, \text { em }[0, T] .
$$

Por fim, combinando (1.3.5) e (1.3.7) temos

$$
g(t) \leqslant 2(a+\varepsilon)^{2}+2 \int_{0}^{t} m(s) d s, \text { em }[0, T] .
$$

Como $\varepsilon>0$ foi tomado arbitrário, a desigualdade (1.3.2) decorre imediatamente de (1.3.8) fazendo $\varepsilon \rightarrow 0$.

Outro resultado a ser utilizado é o teorema de Lax-Milgram. Logo, a definição de forma bilinear, contínua e coerciva definida em um espaço de Hilbert $\mathrm{H}$ se faz necessária. Com efeito, diremos que uma aplicação bilinear $b: \mathrm{H} \times \mathrm{H} \rightarrow \mathbb{R}$ é contínua se existir $\alpha>0$, constante real, para a qual temos

$$
|b(u, v)| \leqslant \alpha\|u\|_{\mathrm{H}}\|v\|_{\mathrm{H}}, \forall u, v \in \mathrm{H}
$$

e coerciva se existir $\beta>0$, constante real, satisfazendo

$$
b(u, u) \geqslant \beta\|u\|_{\mathrm{H}}^{2}, \forall u \in \mathrm{H} .
$$

Segue portanto o resultado.

Proposição 1.3.2 (Lax-Milgram). Sejam $H$ um espaço de Hilbert e $b: H \times H \rightarrow \mathbb{R}$ uma forma bilinear, contínua e coerciva. Então, para todo $v \in H^{\prime}$ existe um único elemento $u \in H$ tal que

$$
b(u, z)=\langle v, z\rangle_{H^{\prime}, H}, \forall z \in H .
$$


Além disso, se b é simétrica então o elemento u é caracterizado por

$$
u \in H \quad e \quad \frac{1}{2} b(u, u)-\langle v, u\rangle_{H^{\prime}, H}=\min _{z \in H}\left\{\frac{1}{2} b(u, z)-\langle v, z\rangle_{H^{\prime}, H}\right\} .
$$

Demonstração. Veja [2] página 84. 


\section{Capítulo 2}

\section{A Equação de Onda Linear}

Neste capítulo formularemos o problema de controlabilidade exata na fronteira para a equação de onda linear e homogêna, bem como resolveremos aplicando o método HUM.

\subsection{Formulação do Problema}

Consideremos uma corda elástica de comprimento $L>0$ e perfeitamente flexível, esticada sobre um eixo $x$. A corda movimenta-se sobre um plano contendo o eixo $x$, de modo que esse movimento seja caracterizado por pequenas oscilações no sentido vertical em relação ao eixo considerado. Suponha ainda que a corda possua extremidade $x=0$ fixa e que a extremidade $x=L$ movimente-se de acordo com uma dada função $v=v(t)$, onde $t$ é a variável representando o instante de observação do movimento. Suponhamos ainda, sem perda de generalidade, que a densidade linear de massa e a tensão em cada ponto da corda sejam constantes, digamos ambas iguais a 1 . Denotando por $u=u(x, t)$ a posição de cada ponto $x$ da corda em cada instante $t$, o princípio de Hamilton fornece a seguinte representação matemática para o fenômeno observado

$$
u_{t t}(x, t)-u_{x x}(x, t)=0, \text { em }(0, L) \times(0, T)
$$

onde $u_{t t}(x, t)=\frac{\partial^{2} u}{\partial t^{2}}(x, t)$ e $u_{x x}(x, t)=\frac{\partial^{2} u}{\partial x^{2}}(x, t)$, e com condições de fronteira

$$
u(0, t)=0, u(L, t)=v(t), \text { em }(0, T) .
$$

Se denotarmos $u_{0}=u_{0}^{0}(x)$ e $u_{1}=u_{0}^{1}(x)$ a posição e a velocidade, respectivamente, de cada ponto $x$ da corda no instante inicial de observação $t=0$, obtemos a condição

$$
u(x, 0)=u_{0}^{0}(x), u_{t}(x, 0)=u_{0}^{1}(x), \text { em }(a, b) .
$$


Com isso, as equações (2.1.1)-(2.1.3) constituem o que se chama problema misto para vibrações transversais de uma corda elástica.

O par $\left\{u_{0}^{0}, u_{0}^{1}\right\}$ constitui o que chamamos configuração incial do sistema (2.1.1)-(2.1.2). De maneira análoga podemos definir configuração em qualquer instante $t \in[0, T]$. Em particular, se $\left\{u_{T}^{0}, u_{T}^{1}\right\}$ é a configuração final então temos

$$
u(x, T)=u_{T}^{0}(x), u_{t}(x, T)=u_{T}^{1}(x), \text { em }(0, L) .
$$

Assim, o problema a ser tratado pode ser formulado da seguinte maneira.

Problema: Para quaisquer configurações inicial e final, a dizer $\left\{u_{0}^{0}, u_{0}^{1}\right\}$ e $\left\{u_{T}^{0}, u_{T}^{1}\right\}$, existe um controle $v$ de modo que o sistema (2.1.1)-(2.1.3) possua uma única solução $u$ satisfazendo a condição adicional (2.1.4).

Observe que a formulação acima é vaga, no sentido de que não foi especificado o espaço onde residem as configurações inicial e final, bem como o espaço onde reside o controle. Além disso, não foi especificado também o conceito de solução a ser adotado para o sistema (2.1.1)-(2.1.3). Assim, as seções que seguem procuram fornecer tais especificações, e também a resolução deste problema através do método HUM.

\subsection{Solução Ultrafraca}

Nesta seção introduziremos o conceito de solução a ser adotado para o problema (2.1.1)-(2.1.3). Porém, são necessário alguns resultados pertinentes ao seguinte problema

$$
\begin{cases}\varphi_{t t}(x, t)-\varphi_{x x}(x, t)=f(x, t), & \text { em }(0, L) \times(0, T) ; \\ \varphi(0, t)=0, \varphi(L, t)=0, & \text { em }(0, T) \\ \varphi(x, 0)=\varphi_{0}(x), \varphi_{t}(x, 0)=\varphi_{1}(x), & \text { em }(0, L)\end{cases}
$$

onde $f, \varphi:[0, L] \times[0, T] \rightarrow \mathbb{R}$ e $\varphi_{0}, \varphi_{1}:[0, L] \rightarrow \mathbb{R}$ são funções dadas. O problema (2.2.1) acima difere do primeiro no que diz respeito a presença de uma força externa $f$ e também pelo fato de as extremidades da corda estarem ambas fixas, veja $(2.2 .1)_{2}$. O problema (2.2.1) é também chamado adjunto, ou homogêneo associado, a (2.1.1)-(2.1.3).

O resultado a seguir fornece a definição de solução fraca para o problema adjunto (2.2.1).

Teorema 2.2.1. Dados $\varphi_{0} \in H_{0}^{1}(0, L), \varphi_{1} \in L^{2}(0, L)$ e $f \in L^{1}\left(0, T ; L^{2}(0, L)\right)$, existe uma única função $\varphi:[0, L] \times[0, T] \rightarrow \mathbb{R}$ satisfazendo 
(i) $\varphi \in L^{\infty}\left(0, T ; H_{0}^{1}(0, L)\right)$;

(ii) $\varphi_{t} \in L^{\infty}\left(0, T ; L^{2}(0, L)\right)$;

(iii) $\frac{d}{d t}\left(\varphi_{t}(t), v\right)+(\varphi(t), v)_{\nabla}=(f(t), v)$, para qualquer $v \in H_{0}^{1}(0, L)$ no sentido de $\mathcal{D}^{\prime}(0, T)$;

(iv) $\varphi_{t t} \in L^{1}\left(0, T ; H^{-1}(0, L)\right)$ e $\varphi_{t t}-\varphi_{x x}=f e m L^{1}\left(0, T ; H^{-1}(0, L)\right)$;

(v) $\varphi(0)=\varphi_{0}$ e $\varphi_{t}(0)=\varphi_{1}$.

Definição 2.2.1 (Solução Fraca). A função $\varphi$ obtida no teorema 2.2.1 recebe o nome de solução fraca para o problema (2.2.1).

Demonstração do teorema 2.2.1. Sejam $\varphi_{0} \in \mathrm{H}_{0}^{1}(0, L), \varphi_{1} \in \mathrm{L}^{2}(0, L)$ e $f \in \mathrm{L}^{1}\left(0, T ; \mathrm{L}^{2}(0, L)\right)$ arbitrários. A demonstração será feita com base no método de Faedo-Galerkin, que consiste em aproximar a função desejada por funções mais regulares. Sendo assim, consideremos a seguinte sequência de funções

$$
\left\{w_{n}(x)=\left(\frac{2}{L}\right)^{\frac{1}{2}} \sin \left[\frac{n \pi}{L} x\right], n \in \mathbb{N}^{*}\right\} .
$$

Esta sequência consiste dos autovetores do operador de Laplace

$$
\Delta \in \mathcal{L}\left(\mathrm{H}_{0}^{1}(0, L) ; \mathrm{H}^{-1}(0, L)\right)
$$

e seus respectivos autovalores $\lambda_{n}=\sqrt{\frac{n \pi}{L}}, n \in \mathbb{N}^{*}$. Além disso, o operador $\Delta$ é um isomorfismo linear de $\mathrm{H}_{0}^{1}(0, L)$ em $\mathrm{H}^{-1}(0, L)$ (veja [10] pág. 304). Também não é difícil ver que neste caso a sequência $\left(w_{n}\right)_{n \in \mathbb{N}^{*}}$ é base ortonormal para o espaço $\left(\mathrm{L}^{2}(0, L),\|\cdot\|\right)$ e, por conseguinte, é base ortogonal para $\left(\mathrm{H}_{0}^{1}(0, L),\|\cdot\|_{\nabla}\right)$.

Seja $m \in \mathbb{N}^{*}$ fixo porém arbitrário. Denotemos por $V_{m}$ o espaço gerado pelos $m$-primeiros elementos da sequência $\left(w_{n}\right)$, isto é, $V_{m}=\operatorname{span}\left\{w_{1}, \ldots, w_{m}\right\}$. Formulamos a seguir o seguinte problema aproximado:

$$
\left\{\begin{array}{l}
\text { encontrar uma função } \varphi^{m}:[0, T] \rightarrow \mathrm{V}_{m} \text { satisfazendo: } \\
\text { (a) }\left(\varphi_{t t}^{m}(t), v\right)+\left(\varphi^{m}(t), v\right)_{\nabla}=(f(t), v), \forall v \in \mathrm{V}_{m} \text { e q.t.p. em }[0, T] ; \\
\text { (b) } \varphi^{m}(0)=\varphi_{0}^{m} ; \varphi_{t}^{m}(0)=\varphi_{1}^{m},
\end{array}\right.
$$

onde $\varphi_{0}^{m}$ e $\varphi_{1}^{m}$ são as aproximações em $V_{m}$ de $\varphi_{0}$ e $\varphi_{1}$, respectivamente. Neste caso, pela escolha da base temos

$$
\varphi_{0}^{m} \rightarrow \varphi_{0} \mathrm{em} \mathrm{H}_{0}^{1}(0, L) \text { e } \varphi_{1}^{m} \rightarrow \varphi_{1}, \mathrm{em} \mathrm{L}^{2}(0, L)
$$


e também são válidas as seguintes expressões

$$
\varphi_{0}^{m}=\sum_{k=1}^{m}\left(\varphi_{0}, \frac{w_{k}}{\sqrt{\lambda_{k}}}\right)_{\nabla} \frac{w_{k}}{\sqrt{\lambda_{k}}}=\sum_{k=1}^{m}\left(\varphi_{0}, w_{k}\right) w_{k} \quad \text { e } \quad \varphi_{1}^{m}=\sum_{k=1}^{m}\left(\varphi_{1}, w_{k}\right) w_{k}
$$

O problema (2.2.2) possui uma única solução, a dizer $\varphi^{m}$, dada por

$$
\varphi^{m}(t)=\sum_{j=1}^{m} g_{m_{j}}(t) w_{j}
$$

onde $\left\{g_{m_{j}}:[0, T] \rightarrow \mathbb{R} ; 1 \leqslant j \leqslant m\right\}$ é solução do sistema de edo's lineares de segunda ordem abaixo

$$
\left\{\begin{array}{l}
g_{m_{j}}^{\prime \prime}(t)+\lambda_{j} g_{m_{j}}(t)=\left(f(t), w_{j}\right) \\
g_{m_{j}}(0)=\left(\varphi_{0}, w_{j}\right) ; g_{m_{j}}^{\prime}(0)=\left(\varphi_{1}, w_{j}\right), \quad 1 \leqslant j \leqslant m
\end{array}\right.
$$

A fórmula da variação das constantes fornece a seguinte expressão para as funções $g_{m_{j}}$ :

$$
g_{m_{j}}(t)=\frac{\left(\varphi_{1}, w_{j}\right)}{\sqrt{\lambda_{j}}} \sin \left[\sqrt{\lambda_{j}} t\right]+\left(\varphi_{0}, w_{j}\right) \cos \left[\sqrt{\lambda_{j}} t\right]+\frac{1}{\sqrt{\lambda_{j}}} \int_{0}^{t}\left(f(s), w_{j}\right) \sin \left[\sqrt{\lambda_{j}}(t-s)\right] d s
$$

para $t \in[0, T]$ e $1 \leqslant j \leqslant m$.

Compondo então (2.2.5) e (2.2.7) obtemos a fórmula explícita para a solução aproximada $\varphi^{m}$ e donde segue a regularidade

$$
\varphi^{m} \in \mathcal{C}\left(0, T ; \mathrm{H}_{0}^{1}(0, L)\right) \subset \mathrm{L}^{\infty}\left(0, T ; \mathrm{H}_{0}^{1}(0, L)\right)
$$

Disto segue que a derivada distribucional vetorial e a derivada usual de $\varphi^{m}$ coincidem. Logo, derivando (2.2.5) e (2.2.7) no sentido usual obtemos

$$
\varphi_{t}^{m}(t)=\sum_{j=1}^{m} g_{m_{j}}^{\prime}(t) w_{j}
$$

com

$$
g_{m_{j}}^{\prime}(t)=\left(\varphi_{1}, w_{j}\right) \cos \left[\sqrt{\lambda_{j}} t\right]-\sqrt{\lambda_{j}} \sin \left[\sqrt{\lambda_{j}} t\right]+\int_{0}^{t}\left(f(s), w_{j}\right) \sin \left[\sqrt{\lambda_{j}}(t-s)\right] d t
$$

para $t \in[0, T]$ e $1 \leqslant j \leqslant m$. Novamente, compondo (2.2.9) e (2.2.10) obtemos a fórmula 
explícita para $\varphi_{t}^{m}$ e também a regularidade

$$
\varphi_{t}^{m} \in \mathcal{C}\left(0, T ; \mathrm{L}^{2}(0, L)\right) \subset \mathrm{L}^{\infty}\left(0, T ; \mathrm{L}^{2}(0, L)\right) .
$$

Contudo, como $m \in \mathbb{N}^{*}$ foi tomado arbitrário concluimos a existência de uma sequência de funções $\left(\varphi^{m}\right)_{m \in \mathbb{N}^{*}}$ satisfazendo o problema (2.2.2) e com regularidades (2.2.8) e (2.2.11).

Vamos mostrar que a sequência $\left(\varphi_{m}\right)_{m \in \mathbb{N}^{*}}$ converge para a função desejada. Para isso, fixemos $m \in \mathbb{N}^{*}$ qualquer. Pelas fórmulas (2.2.9) e (2.2.10) vemos facilmente que $\varphi_{t}^{m}(t) \in V_{m}$ para todo $t \in[0, T]$. Logo, tomando $v=\varphi_{t}^{m}(t)$ em (2.2.2)(a) obtemos a identidade

$$
\frac{d}{d t} \frac{1}{2}\left\{\left\|\varphi^{m}(t)\right\|_{\nabla}^{2}+\left\|\varphi_{t}^{m}(t)\right\|^{2}\right\}=\left(f(t), \varphi_{t}^{m}(t)\right), \text { em q.t.p. de }[0, T] .
$$

Integrando esta última igualdade de 0 a $t \leqslant T$ obtemos

$$
\frac{1}{2}\left\{\left\|\varphi^{m}(t)\right\|_{\nabla}^{2}+\left\|\varphi_{t}^{m}(t)\right\|^{2}\right\}=\frac{1}{2}\left\{\left\|\varphi_{0}^{m}\right\|_{\nabla}^{2}+\left\|\varphi_{1}^{m}\right\|^{2}\right\}+\int_{0}^{t}\left(f(s), \varphi_{t}^{m}(s)\right) d s \text {, em q.t.p. de [0,T]. }
$$

Esta fórmula descreve a energia associada a solução aproximada $\varphi^{m}$.

Utilizando a desigualdade de Cauchy-Schwartz e majorações claras chegamos à desigualdade

$$
\frac{1}{2}\left\{\left\|\varphi^{m}(t)\right\|_{\nabla}+\left\|\varphi_{t}^{m}(t)\right\|\right\}^{2} \leqslant 2\left\{\left\|\varphi_{0}^{m}\right\|_{\nabla}+\left\|\varphi_{1}^{m}\right\|\right\}^{2}+2 \int_{0}^{t}\|f(s)\|\left\{\left\|\varphi_{t}^{m}(s)\right\|+\|\varphi(s)\|_{\nabla}\right\} d s .
$$

Aplicando então a proposição $1.3 .1 \operatorname{com} g(t)=\left\|\varphi^{m}(t)\right\|_{\nabla}+\left\|\varphi_{t}^{m}(t)\right\|, m(s)=\|f(s)\| \mathrm{e}$ $a=\left\|\varphi_{0}^{m}\right\|_{\nabla}+\left\|\varphi_{1}^{m}\right\|$ obtemos a desigualdade

$$
\left\|\varphi^{m}(t)\right\|_{\nabla}+\left\|\varphi_{t}^{m}(t)\right\| \leqslant 2\left[\left\|\varphi_{0}^{m}\right\|_{\nabla}+\left\|\varphi_{1}^{m}\right\|+\int_{0}^{t}\|f(s)\| d s\right], \text { em }[0, T],
$$

donde, pela convergência (2.2.3) somada à desigualdade de Bessel, resulta

$$
\left\|\varphi^{m}(t)\right\|_{\nabla}+\left\|\varphi_{t}^{m}(t)\right\| \leqslant 2\left[\left\|\varphi_{0}\right\|_{\nabla}+\left\|\varphi_{1}\right\|+\|f\|_{L^{1}\left(0, T ; L^{2}(0, L)\right)}\right]
$$

para todo $t \in[0, T]$ e para todo $m \in \mathbb{N}^{*}$. Disto segue que

$$
\left\|\varphi^{m}\right\|_{\mathrm{L}^{\infty}\left(0, T ; \mathrm{H}_{0}^{1}(0, L)\right)}+\left\|\varphi_{t}^{m}\right\|_{\mathrm{L}^{\infty}\left(0, T ; \mathrm{L}^{2}(0, L)\right)} \leqslant 4\left[\left\|\varphi_{0}\right\|_{\nabla}+\left\|\varphi_{1}\right\|+\|f\|_{\mathrm{L}^{1}\left(0, T ; \mathrm{L}^{2}(0, L)\right)}\right]
$$

ou seja, as sequências $\left(\varphi^{m}\right)_{m \in \mathbb{N}^{*}}$ e $\left(\varphi_{t}^{m}\right)_{m \in \mathbb{N}^{*}}$ são limitadas em $\mathbf{L}^{\infty}\left(0, T ; \mathrm{H}_{0}^{1}(0, L)\right)$ e $\mathbf{L}^{\infty}\left(0, T ; \mathbf{L}^{2}(0, L)\right)$, respectivamente. 
Aplicando em seguida o teorema de Banach-Alaoglou-Bourbaki (veja [2] página 42) existem uma subsequência de $\left(\varphi^{m}\right)$, a qual continuaremos denotando por $\left(\varphi^{m}\right)$, e uma função $\varphi \in$ $\mathrm{L}^{\infty}\left(0, T ; \mathrm{H}_{0}^{1}(0, L)\right)$ tais que

$$
\begin{aligned}
& \varphi^{m} \stackrel{*}{\rightarrow} \varphi, \text { em L } \mathrm{L}^{\infty}\left(0, T ; \mathrm{H}_{0}^{1}(0, L)\right)=\left[\mathrm{L}^{1}\left(0, T ; \mathrm{H}^{-1}(0, L)\right)\right]^{\prime} ; \\
& \varphi_{t}^{m} \stackrel{*}{\rightarrow} \varphi_{t}, \quad \mathrm{em} \mathrm{L}^{\infty}\left(0, T ; \mathrm{L}^{2}(0, L)\right)=\left[\mathrm{L}^{1}\left(0, T ; \mathrm{L}^{2}(0, L)\right)\right]^{\prime}
\end{aligned}
$$

onde o sinal " $\stackrel{*}{\rightarrow}$ representa a convergência weak-star para os respectivos duais. Com isto, temos provado $(i)$ e $(i i)$.

A fim de provarmos $(i i i)$, observemos que a convergência (2.2.18) significa que para cada $w \in \mathrm{L}^{1}\left(0, T ; \mathrm{H}^{-1}(0, L)\right)$ temos

$$
\left\langle\varphi^{m}, w\right\rangle_{-1,1}=\int_{0}^{T}\left\langle\varphi^{m}(t), w(t)\right\rangle_{-1,1} d t \rightarrow \int_{0}^{T}\langle\varphi(t), w(t)\rangle_{-1,1} d t=\langle\varphi, w\rangle_{-1,1}
$$

onde $\langle\cdot, \cdot\rangle_{-1,1}$ denota tanto a dualidade entre os espaços $\mathrm{H}_{0}^{1}(0, L)$ e $\mathrm{H}^{-1}(0, L)$ como a dualidade entre os espaços $\mathrm{L}^{\infty}\left(0, T ; \mathrm{H}_{0}^{1}(0, L)\right)$ e $\mathrm{L}^{1}\left(0, T ; \mathrm{H}^{1}(0, L)\right)$. Em particular, para $w=v \theta$, onde $v \in \mathrm{H}_{0}^{1}(0, L)$ e $\theta \in \mathcal{D}(0, T)$ temos $w \in \mathrm{L}^{1}\left(0, T ; \mathrm{H}_{0}^{1}(0, L)\right)$ e $\left\langle w(t), \varphi^{m}(t)\right\rangle_{-1,1}=\left(\varphi^{m}(t), w(t)\right)_{\nabla}$, bem como $\langle w(t), \varphi(t)\rangle_{-1,1}=(\varphi(t), w(t))_{\nabla}$. Nestas condições, (2.2.20) fica

$$
\int_{0}^{T}\left(\varphi^{m}(t), v\right)_{\nabla} \theta(t) d t \rightarrow \int_{0}^{T}(\varphi(t), v)_{\nabla} \theta(t) d t
$$

Analogamente, a convergência (2.2.19) implica

$$
\int_{0}^{T}\left(\varphi_{t}^{m}(t), v\right) \theta(t) d t \rightarrow \int_{0}^{T}\left(\varphi_{t}^{m}(t), v\right) \theta(t) d t
$$

para todo $\theta \in \mathcal{D}(0, T)$ e todo $v \in \mathrm{H}_{0}^{1}(0, L)$, ou seja, para cada $v \in \mathrm{H}_{0}^{1}(0, L)$ temos $\left(\varphi_{t}^{m}(\cdot), v\right) \rightarrow$ $\left(\varphi_{t}(\cdot), v\right)$ no sentido de distribuições reais no intervalo $[0, T]$. Pela continuidade da operação de derivação em $\mathcal{D}^{\prime}(0, T)$ concluímos

$$
\int_{0}^{T}\left(\varphi_{t t}^{m}(t), v\right) \theta(t) d t \rightarrow \int_{0}^{T} \frac{d}{d t}\left(\varphi_{t}(t), v\right) \theta(t) d t
$$

para todo $\theta \in \mathcal{D}(0, T)$ e todo $v \in \mathrm{H}_{0}^{1}(0, L)$.

Assim, fixando $m \in \mathbb{N}^{*}$ e fazendo $n>m \operatorname{com} n \rightarrow \infty$ segue de (2.2.2)(a), (2.2.21) e (2.2.23) 
a identidade

$$
\int_{0}^{T} \frac{d}{d t}\left(\varphi_{t}(t), v\right) \theta(t) d t+\int_{0}^{T}(\varphi(t), v)_{\nabla} \theta(t) d t=\int_{0}^{T}(f(t), v) d t
$$

para todo $v \in V_{m}$ e todo $\theta \in \mathcal{D}(0, T)$. Agora, sendo $\left(w_{n}\right)_{n \in \mathbb{N}^{*}}$ base ortogonal para $\mathrm{H}_{0}^{1}(0, L)$ concluímos que (2.2.24) é válida para todo $v \in \mathrm{H}_{0}^{1}(0, L)$ e todo $\theta \in \mathcal{D}(0, T)$. Disto segue portanto a afirmação $(i i i)$.

Provemos agora a afirmação $(i v)$. De (2.2.24) temos

$$
-\int_{0}^{T}\left(\varphi_{t}(t), v\right) \theta^{\prime}(t) d t=\int_{0}^{T}\left\langle f(t)+\varphi_{x x}(t), v\right\rangle_{-1,1} \theta(t) d t
$$

para todo $v \in \mathrm{H}_{0}^{1}(0, L)$ e todo $\theta \in \mathcal{D}(0, T)$. Denotando $g=f+\varphi_{x x}$ temos $g \in \mathrm{L}^{1}\left(0, T ; \mathrm{H}^{-1}(0, L)\right)$. Logo, de (2.2.25) temos

$$
\left(-\int_{0}^{T} \varphi_{t}(t) \theta^{\prime}(t) d t, v\right)=\left\langle\int_{0}^{T} g(t) \theta(t) d t, v\right\rangle_{-1,1}
$$

para todo $\theta \in \mathcal{D}(0, T)$ e todo $v \in \mathrm{H}_{0}^{1}(0, L)$. Disto segue que

$$
-\int_{0}^{T} \varphi_{t}(t) \theta^{\prime}(t) d t=\int_{0}^{T} g(t) \theta(t) d t, \mathrm{em} \mathrm{H}^{-1}(0, L)
$$

para todo $\theta \in \mathcal{D}(0, T)$, ou seja, $g$ é a derivada distribucional vetorial de $\varphi_{t}$. Denotando esta derivada por $\varphi_{t t}$ a afirmação $(i v)$ segue.

Provemos agora $(v)$. Primeiramente, observemos que como $\varphi \in \mathrm{L}^{1}\left(0, T ; \mathrm{H}_{0}^{1}(0, L)\right)$ e $\varphi_{t} \in$ $\mathrm{L}^{1}\left(0, T ; \mathrm{L}^{2}(0, L)\right)$ segue que $\varphi \in \mathcal{C}\left(0, T ; \mathrm{H}_{0}^{1}(0, L)\right)$ (ver [9] página 11). Analogamente, como $\varphi_{t} \in \mathrm{L}^{1}\left(0, T ; \mathrm{L}^{2}(0, L)\right)$ e $\varphi_{t t} \in \mathrm{L}^{1}\left(0, T ; \mathrm{H}^{-1}(0, L)\right)$ segue que $\varphi_{t} \in \mathcal{C}\left(0, T ; \mathrm{L}^{2}(0, L)\right)$. Logo, faz sentido calcularmos $\varphi(0)$ bem como $\varphi_{t}(0)$.

Para $\varphi(0)$ seja $m \in \mathbb{N}^{*}$ fixo porém arbitrário. Tomando $w=w_{m} \theta^{\prime}$, onde $\theta \in \mathcal{C}^{1}(0, T)$ com $\theta(0)=1$ e $\theta(T)=0$ segue da convergência (2.2.18) o seguinte

$$
\int_{0}^{T}\left(\varphi^{n}(t), w_{m}\right) \theta^{\prime}(t) d t \rightarrow \int_{0}^{T}\left(\varphi(t), w_{m}\right) \theta^{\prime}(t) d t, \text { quando } n \rightarrow \infty
$$

Já por (2.2.19) temos

$$
\int_{0}^{T}\left(\varphi_{t}^{n}(t), w_{m}\right) \theta(t) d t \rightarrow \int_{0}^{T}\left(\varphi_{t}(t), w_{m}\right) \theta(t) d t, \text { quando } n \rightarrow \infty
$$


Agora, como $\frac{d}{d t}\left(\varphi^{n}(\cdot), w_{m}\right)=\left(\varphi_{t}^{n}(\cdot), w_{m}\right)$ decorre de $(2.2 .28)$ e $(2.2 .29)$ o seguinte

$$
\int_{0}^{T} \frac{d}{d t}\left[\left(\varphi^{n}(t), w_{m}\right) \theta(t)\right] d t \rightarrow \int_{0}^{T} \frac{d}{d t}\left[\left(\varphi(t), w_{m}\right) \theta(t)\right] d t
$$

ou ainda $\left(\varphi^{n}(0), w_{n}\right) \rightarrow\left(\varphi(0), w_{m}\right)$ para todo $m \in \mathbb{N}^{*}$. Por outro lado temos $\left(\varphi^{n}(0), w_{m}\right)=$ $\left(\varphi_{0}^{n}, w_{m}\right)=\left(\varphi_{0}, w_{m}\right)$. Portanto $\left(\varphi(0), w_{m}\right)=\left(\varphi_{0}, w_{m}\right)$ para todo $m \in \mathbb{N}^{*}$, donde segue que $\varphi(0)=\varphi_{0} \mathrm{em} \mathrm{H}_{0}^{1}(0, L)$.

Para $\varphi_{t}(0)$, seja $\delta>0$ e defina

$$
\theta_{\delta}(t)=\left\{\begin{array}{cl}
-\frac{1}{\delta} & , \text { se } 0 \leqslant t \leqslant \delta \\
0 & , \text { se } \delta<t \leqslant T
\end{array}\right.
$$

que pertence a $\mathbf{H}^{1}(0, T)$. Multiplicando em seguida $(2.2 .2)(\mathrm{a})$ por $\theta_{\delta}$ e integrando em $[0, T]$ obtemos

$$
\int_{0}^{\delta}\left(\varphi_{t t}^{m}(t), v\right) \theta_{\delta}(t) d t+\int_{0}^{\delta}\left(\varphi^{m}(t), v\right)_{\nabla} \theta_{\delta}(t) d t=\int_{0}^{\delta}(f(t), v) \theta_{\delta}(t) d t
$$

Utilizando integração por partes na primeira integral do primeiro membro de (2.2.32) obtemos

$$
-\left(\varphi_{1}^{m}, v\right)+\frac{1}{\delta} \int_{0}^{\delta}\left(\varphi_{t}^{m}(t), v\right) d t+\int_{0}^{\delta}\left(\varphi^{m}(t), v\right)_{\nabla} \theta_{\delta}(t) d t=\int_{0}^{\delta}(f(t), v) \theta_{\delta}(t) d t
$$

Fazendo então $m \rightarrow \infty$ em (2.2.33) vem

$$
-\left(\varphi_{1}, v\right)+\frac{1}{\delta} \int_{0}^{\delta}(\varphi(t), v) d t+\int_{0}^{\delta}(\varphi(t), v)_{\nabla} \theta_{\delta}(t) d t=\int_{0}^{\delta}(f(t), v) \theta_{\delta}(t) d t
$$

para todo $v \in \mathrm{H}_{0}^{1}(0, L)$. Por outro lado, utilizando o Teorema Fundamental do Cálculo temos

$$
\begin{aligned}
\frac{1}{\delta} \int_{0}^{\delta}\left(\varphi_{t}(t), v\right) d t & =-\int_{0}^{\delta}\left(\varphi_{t}(t), v\right) \theta_{\delta}^{\prime}(t) d t \\
& =-\int_{0}^{\delta} \frac{d}{d t}\left[\left(\varphi_{t}(t), v\right) \theta_{\delta}(t)\right] d t+\int_{0}^{\delta} \frac{d}{d t}\left(\varphi_{t}(t), v\right) \theta_{\delta}(t) d t \\
& =\left(\varphi_{t}(1), v\right)+\int_{0}^{\delta} \frac{d}{d t}\left(\varphi_{t}(t), v\right) \theta_{\delta}(t) d t
\end{aligned}
$$

Substituindo (2.2.35) em (2.2.34) e fazendo $\delta \rightarrow 0$ obtemos

$$
\left(\varphi_{t}(0), v\right)=\left(\varphi_{1}, v\right)
$$


para todo $v \in \mathrm{H}_{0}^{1}(0, L)$. Logo $\varphi_{t}(0)=\varphi_{1}$, como queríamos provar.

Por fim, resta provarmos a unicidade. Seja então $\psi:[0, L] \times[0, T] \rightarrow \mathbb{R}$ uma outra função satisfazendo $(i)-(v)$. Vamos mostrar que $\psi \equiv \varphi$. Defina $z=\varphi-\psi$. A função $z$ satisfaz

$\left(i^{\prime}\right) z \in \mathrm{L}^{\infty}\left(0, T ; \mathrm{H}_{0}^{1}(0, L)\right)$

$\left(i i^{\prime}\right) z_{t} \in \mathrm{L}^{\infty}\left(0, T ; \mathrm{L}^{2}(0, L)\right)$

(iii) $\frac{d}{d t}\left(z_{t}(t), v\right)+(z(t), v)_{\nabla}=0$, para qualquer $v \in \mathrm{H}_{0}^{1}(0, L)$ no sentido de $\mathcal{D}^{\prime}(0, T)$;

(iv) $z_{t t} \in \mathrm{L}^{1}\left(0, T ; \mathrm{H}^{-1}(0, L)\right)$ e $z_{t t}-z_{x x}=0 \mathrm{em} \mathrm{L} \mathrm{L}^{1}\left(0, T ; \mathrm{H}^{-1}(0, L)\right)$

(v) $z(0)=0$ e $z_{t}(0)=0$

Vamos mostrar que nestas condições devemos ter $z \equiv 0$, donde seguirá o resultado.

Para isto, seja $s \in(0, T)$ e defina $\zeta:[0, T] \rightarrow \mathrm{H}_{0}^{1}(0, L)$ por

$$
\zeta(t)=\left\{\begin{array}{cc}
-\int_{t}^{s} z(\tau) d \tau, & \text { se } 0 \leqslant t<s \\
0, & \text { se } s \leqslant t<T
\end{array}\right.
$$

Note que $\zeta \in \mathcal{C}^{1}\left(0, T ; \mathrm{H}_{0}^{1}(0, L)\right) \subset \mathrm{L}^{\infty}\left(0, T ; \mathrm{H}_{0}^{1}(0, L)\right)$ e também $\zeta_{t}(t)=z(t)$ para todo $t \in(0, s)$. Logo, utilizando integração por partes temos

$$
\begin{aligned}
0 & =\int_{0}^{T}\left\langle\zeta(t), z_{t t}(t)-z_{x x}(t)\right\rangle_{-1,1} d t \\
& =\int_{0}^{s}\left\langle\zeta(t), z_{t t}(t)\right\rangle_{-1,1} d t+\int_{0}^{s}(\zeta(t), z(t))_{\nabla} d t \\
& =\int_{0}^{s} \frac{d}{d t}\left(\zeta(t), z_{t}(t)\right) d t-\int_{0}^{s}\left(\zeta_{t}(t), z_{t}(t)\right) d t+\int_{0}^{s}(\zeta(t), z(t))_{\nabla} d t \\
& =\left(\zeta(s), z_{t}(s)\right)-\left(\zeta(0), z_{t}(0)\right)-\int_{0}^{s}\left(z(t), z_{t}(t)\right) d t+\int_{0}^{s}\left(\zeta(t), \zeta_{t}(t)\right)_{\nabla} d t \\
& =\frac{1}{2} \int_{0}^{s}\left\{\|\zeta(t)\|_{\nabla}^{2}-\|z(t)\|^{2}\right\} d t \\
& =-\frac{1}{2}\left\{\|z(s)\|^{2}+\|\zeta(0)\|_{\nabla}^{2}\right\},
\end{aligned}
$$

donde segue que $z(s)=0$. Como $s$ foi escolhido arbitrariamente concluímos que $z \equiv 0$ pois $z$ é contínua. Isto conclui a demonstração do teorema.

Corolário 2.2.2. Nas condições do teorema (2.2.1), a solução fraca $\varphi$ possui a seguinte regularidade

$$
\varphi \in \mathcal{C}\left(0, T ; H_{0}^{1}(0, L)\right) \cap \mathcal{C}^{1}\left(0, T ; L^{2}(0, L)\right)
$$


Corolário 2.2.3. A aplicação linear

$$
\begin{aligned}
\left\{\varphi_{0}, \varphi_{1}, f\right\} & \in H_{0}^{1}(0, L) \times L^{2}(0 . L) \times L^{1}\left(0, T ; L^{2}(0, L)\right) \\
& \mapsto\left\{\varphi, \varphi_{t}\right\} \in L^{\infty}\left(0, T ; H_{0}^{1}(0, L)\right) \times L^{\infty}\left(0, T ; L^{2}(0, L)\right)
\end{aligned}
$$

é contínua.

Lema 2.2.4 (Identidade Fundamental). Para qualquer solução fraca de (2.2.1), digamos por exemplo $\varphi$, é válida a identidade

$$
\begin{aligned}
\frac{1}{2} \int_{0}^{T} h(L)\left|\varphi_{x}(L, t)\right|^{2} d t=\left.\left(h \varphi_{x}(t), \varphi_{t}(t)\right)\right|_{0} ^{T}+\frac{1}{2} \int_{0}^{T} & \int_{0}^{L} h_{x}(x)\left\{\varphi_{x}(x, t)^{2}+\varphi_{t}(x, t)^{2}\right\} d x d t \\
& -\int_{0}^{T} \int_{0}^{L} h(x) \varphi_{x}(x, t) f(x, t) d x d t
\end{aligned}
$$

para toda função $h \in \mathcal{C}^{1}([0, L]), \operatorname{com} h(0)=0$.

Demonstração. Seja $\left\{\varphi_{0}, \varphi_{1}, f\right\} \in \mathcal{D}(0, L) \times \mathcal{D}(0, L) \times \mathcal{D}((0, L) \times(0, T))$. Pelo teorema (2.2.1) existe uma única solução fraca, a dizer $\varphi$, para o problema (2.2.1). Ora, devido a regularidade do dado inicial podemos concluir que de fato temos $\varphi$ solução clássica para (2.2.1). Assim, $\varphi$ satisfaz $(2.2 .1)_{1}$ pontualmente. Logo, para qualquer $h \in \mathcal{C}^{1}([0, L])$ segue que multiplicando $(2.2 .1)_{1}$ por $h$ e integrando em $(0, L) \times(0, T)$ obtemos

$$
\iint_{(0, L) \times(0, T)} h(x) \varphi_{t t}(x, t) d x d t-\iint_{(0, L) \times(0, T)} h(x) \varphi_{x x}(x, t) d x d t=\iint_{(0, L) \times(0, T)} h(x) f(x, t) d x d t .
$$

Utilizando integração por partes em cada uma das integrais no primeiro membro de (2.2.42) obtemos

$$
\iint_{(0, L) \times(0, T)} h(x) \varphi_{t t}(x, t) d x d t=\left.\left(h \varphi_{x}(t), \varphi_{t}(t)\right)\right|_{0} ^{T}+\frac{1}{2} \underset{(0, L) \times(0, T)}{\iint_{x}} h_{x}(x)\left|\varphi_{t}(x, t)\right|^{2} d x d t
$$

e

$$
\iint_{(0, L) \times(0, T)} h(x) \varphi_{x x}(x, t) d x d t=\frac{1}{2} \int_{0}^{T} h(L)\left|\varphi_{x}(L, t)\right|^{2} d t-\frac{1}{2} \underset{(0, L) \times(0, T)}{\iint_{x}} h_{x}(x)\left|\varphi_{x}(x, t)\right|^{2} d x d t .
$$

Substituindo (2.2.43) e (2.2.44) em (2.2.42) obtemos a identidade (2.2.41) para a solução clássica 
$\varphi$. Além disso temos

$$
\begin{aligned}
& \left.\left(h \varphi_{x}(t), \varphi_{t}(t)\right)\right|_{0} ^{T} \leqslant 2 \sup _{t \in[0, T]}\left(h \varphi_{x}(t), \varphi_{t}(t)\right) \\
& \leqslant 2 \max _{x \in[0, L]}|h(x)| \sup _{t \in[0, T]}\|\varphi(t)\|_{\nabla} \sup _{t \in[0, T]}\left\|\varphi_{t}(t)\right\| \\
& \leqslant 2 M\|\varphi\|_{L^{\infty}\left(0, T ; \mathrm{H}_{0}^{1}(0, L)\right)}\left\|\varphi_{t}\right\|_{L^{\infty}\left(0, T ; \mathrm{H}^{2}(0, L)\right)} \\
& \leqslant M\left\{\|\varphi\|_{\mathrm{L}^{\infty}\left(0, T ; \mathrm{H}_{0}^{1}(0, L)\right)}+\left\|\varphi_{t}\right\|_{\mathrm{L}^{\infty}\left(0, T ; \mathrm{H}^{2}(0, L)\right)}\right\}^{2} \\
& \leqslant M C^{2}\left\{\left\|\varphi_{0}\right\|_{\mathrm{H}_{0}^{1}(0, L)}+\left\|\varphi_{1}\right\|_{\mathrm{L}^{2}(0, L)}+\|f\|_{\mathrm{L}^{1}\left(0, T ; \mathrm{L}^{2}(0, L)\right)}\right\}^{2} \text {, }
\end{aligned}
$$

bem como

$$
\begin{aligned}
\iint_{(0, L) \times(0, T)} h_{x}(x)\left|\varphi_{x}(x, t)\right|^{2} d x d t & \leqslant \int_{0}^{T} \int_{0}^{L}\left|h_{x}(x)\right|\left\{\left|\varphi_{x}(x, t)\right|^{2}+\left|\varphi_{x}(x, t)\right|^{2}\right\} d x d t \\
& \leqslant M \int_{0}^{T}\left\{\|\varphi(x)\|_{\nabla}^{2}+\left\|\varphi_{t}(t)\right\|^{2}\right\} d t \\
& \leqslant M\left\{\|\varphi\|_{\mathrm{L}^{\infty}\left(0, T ; \mathrm{H}_{0}^{1}(0, L)\right)}+\left\|\varphi_{t}\right\|_{\mathrm{L}^{\infty}\left(0, T ; \mathrm{L}^{2}(0, L)\right)}\right\}^{2} \\
& \leqslant M C^{2}\left\{\left\|\varphi_{0}\right\|_{\mathrm{H}_{0}^{1}(0, L)}+\left\|\varphi_{1}\right\|_{\mathrm{L}^{2}(0, L)}+\|f\|_{\mathrm{L}^{1}\left(0, T ; \mathrm{L}^{2}(0, L)\right)}\right\}^{2}
\end{aligned}
$$

onde $M \geqslant \max _{x \in[0, L]}|h(x)|+\max _{x \in[0, L]}\left|h_{x}(x)\right|$ e $C=C(L, T)>0$ é constante obtida através da aplicação do corolário 2.2.3. Ainda

$$
\begin{aligned}
\iint_{(0, L) \times(0, T)} h(x) f(x, t) d x d t & \leqslant \int_{0}^{T} \int_{0}^{L}|h(x)| f(x, t) \varphi_{x}(x, t) d x d t \\
& \leqslant M \int_{0}^{T}\left(f(t), \varphi_{x}(t)\right) d t \\
& \leqslant M \int_{0}^{T}\|f(t)\|\left\|\varphi_{x}(t)\right\| d t \\
& \leqslant M \int_{0}^{T}\|f\|\left\{\|\varphi(t)\|_{\nabla}+\left\|\varphi_{t}(t)\right\|\right\} d t \\
& \leqslant M \int_{0}^{T}\|f(t)\| d t\left\{\|\varphi\|_{\mathrm{L} \infty\left(0, T ; \mathrm{H}_{0}^{1}(0, L)\right)}+\left\|\varphi_{t}\right\|_{\mathrm{L} \infty\left(0, T ; \mathrm{L}^{2}(0, L)\right)}\right\} \\
& \leqslant M C\left\{\left\|\varphi_{0}\right\|_{\nabla}+\left\|\varphi_{1}\right\|+\|f\|_{\mathrm{L}^{1}\left(0, T ; \mathrm{L}^{2}(0, L)\right)}\right\}^{2},
\end{aligned}
$$

onde $M$ e $C$ são constantes determinadas como acima. 
Combinando (2.2.45), (2.2.46), (2.2.47) e (2.2.41) obtemos a majoração

$$
\int_{0}^{T}\left|\varphi_{x}(L, t)\right|^{2} d t \leqslant K\left\{\left\|\varphi_{0}\right\|_{\nabla}+\left\|\varphi_{1}\right\|+\|f\|_{\mathrm{L}^{1}\left(0, T ; \mathrm{L}^{2}(0, L)\right)}\right\}^{2}
$$

onde $K=K(M, C)>0$ é constante.

Suponha agora que $\left\{\varphi_{0}, \varphi_{1}, f\right\} \in \mathrm{H}_{0}^{1}(0, L) \times \mathrm{L}^{2}(0, L) \times \mathrm{L}^{1}\left(0, T ; \mathrm{L}^{2}(0, L)\right.$. Por densidade podemos aproximar cada uma das funções dadas por funções testes nos respectivos espaços, ou seja, existe ao menos uma sequência $\left\{\varphi_{0}^{m}, \varphi_{1}^{m}, f^{m}\right\}_{m \in \mathbb{N}^{*}} \in \mathcal{D}(0, L) \times \mathcal{D}(0, L) \times \mathcal{D}((0, L) \times(0, T))$ tal que

$$
\left\{\begin{aligned}
\varphi_{0}^{m} & \rightarrow \varphi_{0} \mathrm{em} \mathrm{H}_{0}^{1}(0, L) \\
\varphi_{1}^{m} & \rightarrow \varphi_{1} \mathrm{em} \mathrm{L}^{2}(0, L) \\
f^{m} & \rightarrow f \mathrm{em} \mathrm{L}^{1}\left(0, T ; \mathrm{L}^{2}(0, L)\right)
\end{aligned}\right.
$$

Considere a sequência $\left(\varphi^{m}\right)_{m \in \mathbb{N}^{*}}$ constituída das soluções clássicas relativa a sequência de dados iniciais $\left\{\varphi_{0}^{m}, \varphi_{1}^{m}, f^{m}\right\}_{m \in \mathbb{N}^{*}}$ satisfazendo (2.2.49). Sejam $m, n \in \mathbb{N}^{*}$ tais que $m>n$. Devido a linearidade do problema (2.2.1) temos $\varphi_{m}-\varphi_{n}$ solução clássica para (2.2.1) bem como a seguinte majoração

$$
\int_{0}^{T}\left|\varphi_{x}^{m}(L, t)-\varphi_{x}^{n}(L, t)\right|^{2} d t \leqslant K\left\{\left\|\varphi_{0}^{m}-\varphi_{0}^{n}\right\|_{\nabla}+\left\|\varphi_{1}^{m}-\varphi_{1}^{n}\right\|+\left\|f^{m}-f^{n}\right\|_{\mathrm{L}^{1}\left(0, T ; \mathbf{L}^{2}(0, L)\right)}\right\}^{2} .
$$

Ora, como para cada $\mu \in \mathbb{N}^{*}$ temos $\varphi^{\mu}(L, \cdot) \in \mathrm{L}^{2}(0, T)$ segue pelas convergências $(2.2 .49)$ e por (2.2.50) que a sequência $\left\{\varphi^{\mu}(L, \cdot)\right\}_{\mu \in \mathbb{N}^{*}}$ é de Cauchy em $L^{2}(0, T)$. Logo, existe um elemento $\Phi \in \mathrm{L}^{2}(0, T)$ para o qual temos $\varphi^{\mu}(L, \cdot) \rightarrow \Phi \mathrm{em} \mathrm{L}^{2}(0, T)$.

Observe que a função $\Phi$ depende do dado inicial $\left\{\varphi_{0}, \varphi_{1}, f\right\}$ mas não depende da escolha da sequência $\left\{\varphi_{0}^{\mu}, \varphi_{1}^{\mu}, f^{\mu}\right\}_{\mu \in \mathbb{N}^{*}}$. Logo, basta definirmos $\varphi_{x}(L, \cdot)=: \Phi$, onde $\varphi$ é a respectiva solução fraca, e o resultado segue.

Corolário 2.2.5 (Traço e Desigualdade Direta). Para qualquer solução fraca de (2.2.1), digamos por exemplo $\varphi$, são válidas as seguintes propriedades

(i) $\varphi_{x}(L, \cdot) \in L^{2}(0, T)$;

(ii) existe $C_{1}=C_{1}(T, L)>0$ constante real tal que

$$
\left\|\varphi_{x}(L, \cdot)\right\|_{L^{2}(0, T)} \leqslant C_{1}\left[\left\|\varphi_{0}\right\|_{H_{0}^{1}(0, L)}+\left\|\varphi_{1}\right\|_{L^{2}(0, L)}+\|f\|_{L^{1}\left(0, T ; H_{0}^{1}(0, L)\right)}\right] .
$$


Temos, portanto, todos os requisitos necessários para introduzir o conceito de solução ultrafraca para (2.1.1)-(2.1.3) de modo a generalizar as noções clássica e fraca de soluções. Este tipo de solução foi concebido por J. L. Lions, veja por exemplo [7] e [3], ao estudar o problema (2.1.1)-(2.1.3) com dados iniciais $u_{0}$ e $u_{1}$ menos regulares que aqueles adotados para soluções clássicas e fracas, ou seja, quando os dados iniciais $u_{0}$ e $u_{1}$ pertencem a $\mathrm{L}^{2}(0, L) \mathrm{e} \mathrm{H}^{-1}(0, L)$, respectivamente.

Primeiramente, reescrevemos o sistema (2.1.1)-(2.1.3) da seguinte forma

$$
\begin{cases}u_{t t}(x, t)-u_{x x}(x, t)=0, & \text { em }(0, L) \times(0, T) \\ u(0, t)=0 ; u(L, t)=v(t), & \text { em }(0, T) \\ u(x, 0)=u_{0}(x) ; u_{t}(x, 0)=u_{1}(x), & \text { em }(0, L)\end{cases}
$$

A seguir, procederemos de maneira heurística a fim de motivar a definição de solução ultrafraca para (2.2.52). De fato, multiplicando ambos os lados da equação $(2.2 .52)_{1}$ por uma função $\varphi=\varphi(x, t)$, com $x \in(0, L)$ e $t \in(0, T)$, integrando por partes em $(0, L) \times(0, T)$ e lembrando as condições $(2.2 .52)_{2}$ e $(2.2 .52)_{3}$ obtemos

$$
\begin{aligned}
0= & \int_{0}^{T} \int_{0}^{L} u\left[\varphi_{t t}-\varphi_{x x}\right] d x d t+\int_{0}^{L}\left\{u_{t}(x, T) \varphi(x, T)-u(x, T) \varphi_{t}(x, T)\right\} d x \\
& -\int_{0}^{L}\left\{u_{1}(x) \varphi(x, 0)-u_{0}(x) \varphi_{t}(x, 0)\right\} d x-\int_{0}^{T}\left\{u_{x}(L, t) \varphi(L, t)-v(t) \varphi_{x}(L, t)\right\} d t \\
& +\int_{0}^{T}\left\{u_{x}(0, t) \varphi(0, t)\right\} d t
\end{aligned}
$$

Observe que nada podemos afirmar sobre os valores $u(x, T), u_{t}(x, T), u_{x}(L, t)$ e $u_{x}(0, t)$. Logo, supondo que $\varphi$ satisfaz

$$
\begin{cases}\varphi_{t t}(x, t)-\varphi_{x x}(x, t)=f(x, t), & \text { em }(0, L) \times(0, T) \\ \varphi(0, t)=0 ; \varphi(L, t)=0, & \text { em }(0, T) \\ \varphi(x, T)=0 ; \varphi_{t}(x, T)=0, & \text { em }(0, L)\end{cases}
$$

a expressão (2.2.53) fica

$$
\int_{0}^{T} \int_{0}^{L} u(x, t) f(x, t) d x d t=\int_{0}^{L} u_{1}(x) \varphi(x, 0) d x-\int_{0}^{L} u_{0}(x) \varphi_{t}(x, 0) d x-\int_{0}^{T} v(t) \varphi_{x}(L, t) d t .
$$


Vimos antes que para cada $f \in \mathrm{L}^{1}\left(0, T ; \mathrm{L}^{2}(0, L)\right)$ o problema (2.2.55) admite uma única solução $\left\{\varphi, \varphi_{t}\right\} \in \mathrm{L}^{\infty}\left(0, T ; \mathrm{H}_{0}^{1}(0, L)\right) \times \mathrm{L}^{\infty}\left(0, T ; \mathrm{L}^{2}(0, L)\right)$. Logo, uma vez que pretendemos tomar $u_{0} \in \mathrm{L}^{2}(0, L)$ e $u_{1} \in \mathrm{H}^{-1}(0, L)$, o lado direito de (2.2.55) fará sentido sempre que $v \in \mathrm{L}^{2}(0, L)$ e motivará a definição de um funcional linear $u$ definido em $\mathrm{L}^{1}\left(0, T ; \mathrm{L}^{2}(0, L)\right)$ o qual adotaremos como solução. Isto motiva a seguinte definição de solução para o problema (2.2.52).

Definição 2.2.2 (Solução Ultrafraca). Dados $u_{0} \in \mathrm{L}^{2}(0, L), u_{1} \in \mathrm{H}^{-1}(0, L)$ e $v \in \mathrm{L}^{2}(0, T)$, dizemos que uma função $u \in \mathrm{L}^{\infty}\left(0, T ; \mathrm{L}^{2}(0, L)\right)$ é uma solução ultrafraca de (2.2.52) se para cada $f \in \mathrm{L}^{1}\left(0, T ; \mathrm{L}^{2}(0, L)\right)$ é válida a seguinte identidade

$$
\int_{0}^{T} \int_{0}^{L} u(x, t) f(x, t) d x d t=\left\langle u_{1}, \varphi(0)\right\rangle_{-1,1}-\left(u_{0}, \varphi_{t}(0)\right)-\int_{0}^{T} v(t) \varphi_{x}(L, t) d t
$$

onde $\varphi$ é a respectiva solução fraca do problema adjunto (2.2.54).

Teorema 2.2.6. Dados $u_{0} \in L^{2}(0, L), u_{1} \in H^{-1}(0, L)$ e $v \in L^{2}(0, T)$, o problema $(2.2 .52)$ admite uma única solução ultrafraca, a dizer u. Além disso, esta solução possui a seguinte regularidade

$$
u \in \mathcal{C}\left(0, T ; L^{2}(0, L)\right) \cap \mathcal{C}^{1}\left(0, T ; H^{-1}(0, L)\right)
$$

Existe ainda uma constante $C=C(T, L)>0$ para a qual temos a desigualdade

$$
\|u\|_{L^{\infty}\left(0, T ; L^{2}(0, L)\right)}+\left\|u_{t}\right\|_{L^{\infty}\left(0, T ; H^{-1}(0, L)\right)} \leqslant C\left[\left\|u_{0}\right\|_{L^{2}(0, L)}+\left\|u_{1}\right\|_{H^{-1}(0, L)}+\|v\|_{L^{2}(0, T)}\right]
$$

Demonstração. Sejam $u_{0} \in \mathrm{L}^{2}(0, L), u_{1} \in \mathrm{H}^{-1}(0, L)$ e $v \in \mathrm{L}^{2}(0, T)$. Definindo a seguinte aplicação $u: \mathrm{L}^{1}\left(0, T ; \mathrm{L}^{2}(0, L)\right) \rightarrow \mathbb{R}$ dada por

$$
u(f)=\left\langle u_{1}, \varphi(0)\right\rangle_{-1,1}-\left(u_{0}, \varphi_{t}(0)\right)-\int_{0}^{T} v(t) \varphi_{x}(L, t) d t, \forall f \in \mathrm{L}^{1}\left(0, T ; \mathrm{L}^{2}(0, L)\right),
$$

onde $\varphi$ é a solução fraca de (2.2.54) correspondente ao dado inicial $\{0,0, f\}$. Esta aplicação está bem definida devido ao teorema 2.2.1. Além disso, do corolário 2.2.3 permite concluir que $u$ é linear. Calculando o valor absoluto temos

$$
\begin{aligned}
|u(f)| & \leqslant\left\|u_{1}\right\|_{\mathrm{H}^{-1}(0, L)}\left\|\varphi_{t}(0)\right\|_{\nabla}+\left\|u_{0}\right\|\|\varphi(0)\|+\|v\|_{\mathrm{L}^{2}(0, T)}\left\|\varphi_{x}(L, \cdot)\right\|_{\mathrm{L}^{2}(0, T)} \\
& \leqslant\left\{\left\|u_{0}\right\|+\left\|u_{1}\right\|_{\mathrm{H}^{-1}(0, L)}\right\}\left\{\|\varphi\|_{\mathrm{L}^{\infty}\left(0, T ; \mathrm{H}_{0}^{1}(0, L)\right)}+\left\|\varphi_{t}\right\|_{\mathrm{L}^{\infty}\left(0, T ; \mathrm{L}^{2}(0, L)\right)}\right\}+\|v\|_{\mathrm{L}^{2}(0, T)}\left\|\varphi_{x}(L, \cdot)\right\|_{\mathrm{L}^{2}(0, T)} \\
& \leqslant C\left\{\left\|u_{0}\right\|+\left\|u_{1}\right\|_{\mathrm{H}^{-1}(0, L)}+\|v\|_{\mathrm{L}^{2}(0, T)}\right\}\|f\|_{\mathrm{L}^{1}\left(0, T ; \mathrm{L}^{2}(0, L)\right)}
\end{aligned}
$$

para toda função $f \in \mathrm{L}^{1}\left(0, T ; \mathrm{L}^{2}(0, L)\right)$, onde $C=C(T, L)>0$ é constante obtida através 
da aplicação do corolário 2.2.3. Isto implica que o funcional linear $u$ é contínuo. Logo, pela proposição 1.2.3 podemos identificar $u$ com um elemento de $\mathrm{L}^{\infty}\left(0, T ; \mathrm{L}^{2}(0, L)\right)$, o qual continuaremos denotando por $u$. Além disso temos

$$
\|u\|_{\mathrm{L}^{\infty}\left(0, T ; \mathrm{L}^{2}(0, L)\right)} \leqslant C\left\{\left\|u_{0}\right\|+\left\|u_{1}\right\|_{\mathrm{H}^{-1}(0, L)}+\|v\|_{\mathrm{L}^{2}(0, T)}\right\}
$$

Note que

$$
u(f)=\langle u, f\rangle_{-1,1}=\int_{0}^{T}\langle u(t), f(t)\rangle_{-1,1} d t=\int_{0}^{T}(u(t), f(t)) d t=\int_{0}^{T} \int_{0}^{L} u(x, t) f(x, t) d x d t .
$$

Segue portanto, que $u$ é uma solução ultrafraca para o problema (2.2.52).

Para a unicidade, suponha que $z$ seja outra solução ultrafraca para o problema (2.2.52) correspondente ao dado inicial $\left\{u_{0}, u_{1}, v\right\}$. Então, temos

$$
\int_{0}^{T} \int_{0}^{L} z f d x d t=\left\langle u_{1}, \varphi_{t}(0)\right\rangle_{-1,1}-\left(u_{0}, \varphi(0)\right)-\int_{0}^{T} v(t) \varphi_{x}(L, t) d t, f \in \mathrm{L}^{1}\left(0, T ; \mathrm{L}^{2}(0, L)\right)
$$

para qualquer $f \in \mathrm{L}^{1}\left(0, T ; \mathrm{L}^{2}(0, L)\right)$. Logo, por (2.2.59) e (2.2.63) obtemos

$$
\langle u-z, f\rangle_{-1,1}=\int_{0}^{T} \int_{0}^{L}(u-z) f d x d t=0,
$$

para todo $f \in \mathrm{L}^{1}\left(0, T ; \mathrm{L}^{2}(0, L)\right)$, em particular para toda $f \in \mathcal{D}((0, L) \times(0, T))$. Assim, aplicando o lema de Du Bois Raymond (ver [4], página 10) segue que $u-z=0$ em $\mathrm{L}^{\infty}\left(0, T ; \mathrm{L}^{2}(0, L)\right)$, donde concluímos a unicidade. Para a regularidade veja [9], página 106.

Corolário 2.2.7. A aplicação linear

$$
\begin{aligned}
\left\{u_{0}, u_{1}, v\right\} & \in L^{2}(0, L) \times H^{-1}(0, L) \times L^{2}(0, T) \\
& \mapsto\left\{u, u_{t}\right\} \in L^{\infty}\left(0, T ; L^{2}(0, L)\right) \times L^{\infty}\left(0, T ; H^{-1}(0, L)\right)
\end{aligned}
$$

onde u é a solução ultrafraca de (2.2.52) correspondente ao dado inicial $\left\{u_{0}, u_{1}, v\right\}$, é contínua.

\subsection{Resolução do Problema}

Nesta seção daremos uma definição matemática para o problema de contolabilidade exata proposto antes e utilizaremos o método HUM para resolvermos esse problema. 
Definição 2.3.1 (Controle Exato). Dado $T>0$, dizemos que o problema (2.2.52) é exatamente controlável no tempo $T$ se existir $v \in \mathrm{L}^{2}(0, T)$ tal que para qualquer dado inicial $\left\{u_{0}, u_{1}\right\} \in$ $\mathrm{L}^{2}(0, L) \times \mathrm{H}^{-1}(0, L)$ o problema (2.2.52) admite uma solução ultrafraca, a dizer $u$, que satisfaz a seguinte condição adicional

$$
u(x, T)=0, u_{t}(x, T)=0, \text { em }(0, L)
$$

Diremos simplesmente que (2.2.52) é exatamente controlável se for controlável para algum $T>0$.

O método HUM, idealizado por J.-L. Lions, que utilizaremos a seguir, consiste na obtenção de um certo critério de unicidade e na construção de um espaço de Hilbert, por completamento, onde obteremos controlabilidade exata para o problema (2.2.52).

A seguir apresentamos o resultado principal deste trabalho.

Teorema 2.3.1. Existe $T_{0}>0$ para o qual o sistema (2.2.52) é exatamente controlável para todo $T>T_{0}$.

Demonstração. Suponha $T>0$ suficientemente grande. Seja o dado inicial $\left\{\varphi_{0}, \varphi_{1}\right\} \in \mathcal{D}(0, T)^{2}$ qualquer. Então, aplicando o teorema 2.2.1, o problema

$$
\begin{cases}\varphi_{t t}(x, t)-\varphi_{x x}=0, & \text { em }(0, L) \times(0, T) \\ \varphi(0, t)=0 ; \varphi(L, t)=0, & \text { em }(0, L) \\ \varphi(x, 0)=\varphi_{0}(x) ; \varphi_{t}(x, 0)=\varphi_{1}(x), & \text { em }(0, L)\end{cases}
$$

admite uma única solução, a dizer $\varphi$. Além disso, do corolário 2.2 .5 temos $\varphi_{x}(L, \cdot) \in \mathrm{L}^{2}(0, T)$. Logo podemos formular o seguinte problema adjunto

$$
\begin{cases}\psi_{t t}(x, t)-\psi_{x x}(x, t)=0, & \text { em }(0, L) \times(0, T) \\ \psi(0, t)=0 ; \psi(L, t)=\varphi_{x}(L, t), & \text { em }(0, T) \\ \psi(x, T)=0 ; \psi_{t}(x, T)=0, & \text { em }(0, L)\end{cases}
$$

Aplicando o teorema 2.2.6 a este problema obteremos uma única solução ultrafraca, a dizer $\psi$. Podemos ainda calcular os instantes iniciais $\left\{\psi(0), \psi_{t}(0)\right\} \in \mathrm{L}^{2}(0, L) \times \mathrm{H}^{-1}(0, L)$. Não é difícil ver que, neste caso, a identidade (2.3.1) fica

$$
\int_{0}^{T}\left|\varphi_{x}(L, t)\right|^{2} d t=\left\langle\psi_{t}(0), \varphi_{1}\right\rangle_{-1,1}-\left(\psi(0), \varphi_{0}\right)
$$


Por outro lado, a unicidade das soluções obtidas e a linearidade assegurada nos corolários 2.2.3 e 2.2.7 permitem concluir que a aplicação

$$
\begin{aligned}
\Lambda: \mathcal{D}(0, L)^{2} & \rightarrow \mathrm{H}^{-1}(0, L) \times \mathrm{L}^{2}(0, L) \\
\left\{\varphi_{0}, \varphi_{1}\right\} & \mapsto \Lambda\left\{\varphi_{0}, \varphi_{1}\right\}=\left\{\psi_{t}(0),-\psi(0)\right\}
\end{aligned}
$$

onde $\psi$ é a correspondente solução ultrafraca do problema (2.3.3), com $\varphi$ solução fraca do problema (2.3.2) correspondente ao dado inicial $\left\{\varphi_{0}, \varphi_{1}\right\}$, é linear.

Considere agora a aplicação

$$
\begin{aligned}
(\cdot, \cdot)_{\mathrm{F}}: \mathcal{D}(0, L)^{2} \times \mathcal{D}(0, L)^{2} & \rightarrow \mathbb{R} \\
\left\{\varphi_{0}, \varphi_{1}\right\},\left\{\xi_{0}, \xi_{1}\right\} & \mapsto\left(\left\{\varphi_{0}, \varphi_{1}\right\},\left\{\xi_{0}, \xi_{1}\right\}\right)_{\mathrm{F}}=\left\langle\Lambda\left\{\varphi_{0}, \varphi_{1}\right\},\left\{\xi_{0}, \xi_{1}\right\}\right\rangle_{-1,1}
\end{aligned}
$$

onde $\langle\cdot, \cdot\rangle_{-1,1}$ denota a dualidade entre os espaços $\mathrm{H}^{-1}(0, L) \times \mathrm{L}^{2}(0, L)$ e $\mathrm{H}_{0}^{1}(0, L) \times \mathrm{L}^{2}(0, L)$.

Considere o seguinte resultado.

Lema 2.3.2 (Desigualdade Inversa). Existe $T_{0}>0$ tal que para cada $T>T_{0}$ podemos encontrar $C_{2}=C_{2}(L, T)>0$, constante, satisfazendo

$$
C_{2}\left\|\left\{\varphi_{0}, \varphi_{1}\right\}_{H}\right\| \leqslant\left\|\varphi_{x}(L, \cdot)\right\|_{L^{2}(0, T)}
$$

para qualquer $\left\{\varphi_{0}, \varphi_{1}\right\} \in H$, com $\varphi$ a respectiva solução fraca do problema (2.3.2).

Com o lema (2.3.2) podemos verificar que $(\cdot, \cdot)_{\mathrm{F}}$ é um produto interno em $\mathcal{D}(0, T)^{2} \times \mathcal{D}(0, T)^{2}$. Logo este define uma norma que será denotada por $\|\cdot\|_{\mathrm{F}}$. Podemos então completar $\mathcal{D}(0, T)^{2}$ com respeito a norma $\|\cdot\|_{F}$ a fim de obtermos um espaço de Hilbert, o qual denotaremos por $F$.

Observe que pelo exposto acima, se $\left\{\varphi_{0}, \varphi_{1}\right\} \in \mathcal{D}(0, L)^{2}$ então temos

$$
\begin{aligned}
\left\|\left\{\varphi_{0}, \varphi_{1}\right\}\right\|_{\mathrm{F}}^{2} & =\left\langle\Lambda\left\{\varphi_{0}, \varphi_{1}\right\},\left\{\varphi_{0}, \varphi_{1}\right\}\right\rangle_{-1,1} \\
& =\left\langle\left\{\psi_{t}(0),-\psi(0)\right\},\left\{\varphi_{0}, \varphi_{1}\right\}\right\rangle_{-1,1} \\
& =\left\langle\psi_{t}(0), \varphi_{0}\right\rangle_{-1,1}-\left(\psi(0), \varphi_{1}\right) \\
& =\int_{0}^{T}\left|\varphi_{x}(L, t)\right|^{2} d t \\
& =\left\|\varphi_{x}(L, \cdot)\right\|_{\mathrm{L}^{2}(0, T)}^{2} \\
& \leqslant C_{1}\left[\left\|\varphi_{0}\right\|_{\mathrm{H}_{0}^{1}(0, L)}^{2}+\left\|\varphi_{1}\right\|_{\mathrm{L}^{2}(0, L)}^{2}\right]=C_{1}\left\|\left\{\varphi_{0}, \varphi_{1}\right\}\right\|_{\mathrm{H}_{0}^{1}(0, L) \times \mathrm{L}^{2}(0, L)}^{2},
\end{aligned}
$$


onde $C_{1}>0$ é uma constante obtida no corolário 2.2.5. Assim, pela densidade de $\mathcal{D}(0, L)^{2} \mathrm{em}$ $\mathrm{H}_{0}^{1}(0, L) \times \mathrm{L}^{2}(0, L)$ podemos concluir de (2.3.8) o seguinte

$$
\mathrm{H}=: \mathrm{H}_{0}^{1}(0, L) \times \mathrm{L}^{2}(0, L) \subset \mathrm{F} .
$$

Ora, pela definição do operador $\Lambda$, a inclusão (2.3.9) permite estender o operador linear $\Lambda$ ao subespaço $\mathrm{H}$ de $\mathrm{F}$.

Desejamos mostrar que o operador $\Lambda: \mathrm{H} \rightarrow \mathrm{H}^{\prime}$ é, de fato, contínuo e sobrejetivo. Primeiramente, observemos que para quaisquer $\left\{\varphi_{0}, \varphi_{1}\right\},\left\{\xi_{0}, \xi_{1}\right\} \in \mathrm{H}$ temos

$$
\left\langle\Lambda\left\{\varphi_{0}, \varphi_{1}\right\},\left\{\xi_{0}, \xi_{1}\right\}\right\rangle_{\mathrm{H}^{\prime}, \mathrm{H}}=\int_{0}^{T} \varphi_{x}(L, t) \xi_{x}(L, T) d t=\left(\varphi_{x}(L, \cdot), \xi_{x}(L, \cdot)\right)_{\mathrm{L}^{2}(0, T)}
$$

onde $\varphi$ e $\xi$ são as soluções fracas de (2.3.2) correspondente aos dados iniciais $\left\{\varphi_{0}, \varphi_{1}\right\}$ e $\left\{\xi_{0}, \xi_{1}\right\}$ respectivamente. Além disso, do corolário 2.2.5 temos

$$
\left|\left\langle\Lambda\left\{\varphi_{0}, \varphi_{1}\right\},\left\{\xi_{0}, \xi_{1}\right\}\right\rangle_{\mathrm{H}^{\prime}, \mathrm{H}}\right| \leqslant C_{1}^{2}\left\|\left\{\varphi_{0}, \varphi_{1}\right\}\right\|_{\mathrm{H}}\left\|\left\{\xi_{0}, \xi_{1}\right\}\right\|_{\mathrm{H}} .
$$

Fixando apenas $\left\{\varphi_{0}, \varphi_{1}\right\}$ na desigualdade acima e lembrando que $\Lambda\left\{\varphi_{0}, \varphi_{1}\right\} \in \mathrm{H}^{\prime}$ chegamos a

$$
\left\|\Lambda\left\{\varphi_{0}, \varphi_{1}\right\}\right\|_{\mathrm{H}^{\prime}} \leqslant C_{1}^{2}\left\|\left\{\varphi_{0}, \varphi_{1}\right\}\right\|_{\mathrm{H}}
$$

Variando agora $\left\{\varphi_{0}, \varphi_{1}\right\}$ em $\mathrm{H}$, e lembrando que a constante $C_{1}>0$ independe de dado $\left\{\varphi_{0}, \varphi_{1}\right\}$ podemos concluir a continuidade do operador $\Lambda$.

Decorre ainda do lema acima e de (2.3.10) a seguinte desigualdade

$$
C_{2}^{2}\left\|\left\{\varphi_{0}, \varphi_{1}\right\}\right\|_{\mathrm{H}}^{2} \leqslant\left|\left\langle\Lambda\left\{\varphi_{0}, \varphi_{1}\right\},\left\{\varphi_{0}, \varphi_{1}\right\}\right\rangle_{\mathrm{H}^{\prime}, \mathrm{H}}\right|
$$

para qualquer $\left\{\varphi_{0}, \varphi_{1}\right\} \in \mathrm{H}$. Disto segue que a aplicação bilinear contínua

$$
\left\{\varphi_{0}, \varphi_{1}\right\},\left\{\xi_{0}, \xi_{1}\right\} \in \mathrm{H} \times \mathrm{H} \mapsto\left\langle\Lambda\left\{\varphi_{0}, \varphi_{1}\right\},\left\{\xi_{0}, \xi_{1}\right\}\right\rangle_{\mathrm{H}^{\prime}, \mathrm{H}}
$$

é coerciva. Aplicando em seguida a proposição 1.3.2 de Lax-Milgram concluímos que para cada $\left\{\eta_{0}, \eta_{1}\right\} \in \mathrm{H}^{\prime}$ existe um único elemento $\left\{\varphi_{0}, \varphi_{1}\right\} \in \mathrm{H}$ para o qual temos

$$
\left\langle\Lambda\left\{\varphi_{0}, \varphi_{1}\right\},\left\{\xi_{0}, \xi_{1}\right\}\right\rangle_{\mathrm{H}^{\prime}, \mathrm{H}}=\left\langle\left\{\eta_{0}, \eta_{1}\right\},\left\{\xi_{0}, \xi_{1}\right\}\right\rangle_{\mathrm{H}^{\prime}, \mathrm{H}}, \forall\left\{\xi_{0}, \xi_{1}\right\} \in \mathrm{H}
$$


donde segue que $\Lambda\left\{\varphi_{0}, \varphi_{1}\right\}=\left\{\eta_{0}, \eta_{1}\right\}$. Logo, segue que o operador $\Lambda: \mathrm{H} \rightarrow \mathrm{H}^{\prime}$ é sobrejetivo.

Contudo, concluímos que $\Lambda: \mathrm{H} \rightarrow \mathrm{H}^{\prime}$ é isomorfismo linear. Assim, para quaisquer $u_{0} \in$ $\mathrm{L}^{2}(0, L)$ e $u_{1} \in \mathrm{H}^{-1}(0, L)$ temos $\left\{u_{1},-u_{0}\right\} \in \mathrm{H}^{\prime}$ e, portanto, existe um único par $\left\{\varphi_{0}, \varphi_{1}\right\} \in \mathrm{H}$ tal que

$$
\Lambda\left\{\varphi_{0}, \varphi_{1}\right\}=\left\{u_{1},-u_{0}\right\}
$$

Por outro lado, pela definição do operador $\Lambda$ temos

$$
\Lambda\left\{\varphi_{0}, \varphi_{1}\right\}=\left\{\psi_{t}(0),-\psi(0)\right\}
$$

onde $\psi$ é a soluão ultrafraca do problema (2.3.3), $\operatorname{com} \varphi$ a solução fraca de (2.3.2) correspondente ao dado inicial $\left\{\varphi_{0}, \varphi_{1}\right\}$. De (2.3.16) e (2.3.17) concluímos

$$
\psi(0)=u_{0} \quad \text { e } \quad \psi_{t}(0)=u_{1}
$$

Entretanto, para $\left\{u_{0}, u_{1}, \varphi_{x}(L, \cdot)\right\} \in \mathrm{L}^{2}(0, L) \times \mathrm{H}_{0}^{1}(0, L) \times \mathrm{L}^{2}(0, T)$ o teorema 2.2.6 garante a existência de uma única solução ultrafraca, a dizer $u$, para o problema (2.2.52). Ora, não é difícil ver que pelas propriedades de linearidade e reversabilidade no tempo temos que $\psi \equiv u$. Logo, a solução $u$ de (2.2.52) satisfaz a condição adicional

$$
u(x, T)=\psi(x, T)=0 \quad \text { e } \quad u_{t}(x, T)=\psi_{t}(x, T)=0 .
$$

Assim, tomando $v=\varphi_{x}(L, \cdot) \in \mathrm{L}^{2}(0, T)$ como controle concluímos, a menos da verificação do lema 2.3.2, que (2.2.52) é exatamente controlável no tempo $T$.

A seguir provaremos o lema 2.3.2 a fim de justificar o resultado anterior.

Demonstração do Lema 2.3.2. Sejam $\left\{\varphi_{0}, \varphi_{1}\right\} \in \mathrm{H}=\mathrm{H}_{0}^{1}(0, L) \times \mathrm{L}^{2}(0, L)$ e $\varphi$ a respectiva solução fraca de (2.3.2). Então, o lema 2.2.4 aplicado a esta solução, com $h=i d_{[0, L]}$ a função identidade em $[0, L]$ fornece

$$
\frac{L}{2} \int_{0}^{T}\left|\varphi_{x}(L, t)\right|^{2} d t=\left.\left(i d_{[0, L]} \varphi_{x}(t), \varphi_{t}(t)\right)\right|_{0} ^{T}+\frac{1}{2} \int_{0}^{T} \int_{0}^{L}\left\{\left|\varphi_{x}(x, t)\right|^{2}+\left|\varphi_{t}(x, t)\right|^{2}\right\} d x d t .(2.3 .20)
$$


Denotando $X=\left.\left(i d_{[0, L]} \varphi_{x}(t), \varphi_{t}(t)\right)\right|_{0} ^{T}$ temos

$$
\begin{aligned}
|X| & =\left|\left(i d_{[0, L]} \varphi_{x}(t), \varphi_{t}(t)\right)\right|_{0}^{T} \mid \\
& \leqslant 2 \sup _{0 \leqslant t \leqslant T}\left|\left(i d_{[0, L]} \varphi_{x}(t), \varphi_{t}(t)\right)\right| \\
& \leqslant 2 L \sup _{t \in[0, T]}\|\varphi(t)\|_{\mathrm{H}_{0}^{1}(0, L)} \sup _{t \in[0, T]}\left\|\varphi_{t}(t)\right\|_{\mathrm{L}^{2}(0, L)} \\
& \leqslant 2 L\|\varphi\|_{\mathrm{L}^{\infty}\left(0, T ; \mathrm{H}_{0}^{1}(0, L)\right)}\left\|\varphi_{t}\right\|_{\mathrm{L}^{\infty}\left(0, T ; \mathrm{L}^{2}(0, L)\right)} \\
& \leqslant L\left\{\|\varphi\|_{\mathrm{L}^{\infty}\left(0, T ; \mathrm{H}_{0}^{1}(0, L)\right)}^{2}+\left\|\varphi_{t}\right\|_{\mathrm{L}^{\infty}\left(0, T ; \mathrm{L}^{2}(0, L)\right)}^{2}\right\} \\
& \leqslant L\left\{\|\varphi\|_{\mathrm{L}^{\infty}\left(0, T ; \mathrm{H}_{0}^{1}(0, L)\right)}+\left\|\varphi_{t}\right\|_{\mathrm{L}^{\infty}\left(0, T ; \mathrm{L}^{2}(0, L)\right)}\right\}^{2} \\
& \leqslant L C^{2}\left\|\left\{\varphi_{0}, \varphi_{1}\right\}\right\|_{\mathrm{H}_{0}^{1}(0, L) \times \mathrm{L}^{2}(0, L)}^{2}
\end{aligned}
$$

onde $C=C(L, T)>0$ é constante obtida aplicando-se o corolário (2.2.3).

Agora, analisando o segundo termo no segundo membro de (2.3.20) temos

$$
\begin{aligned}
& \int_{0}^{T} \int_{0}^{L}\left|\varphi_{x}(x, t)\right|^{2}+\left|\varphi_{t}(x, t)\right|^{2} d x d t=\int_{0}^{T}\left\{\|\varphi(t)\|_{\mathrm{H}_{0}^{1}(0, L)^{2}}+\left\|\varphi_{t}(t)\right\|_{\mathrm{L}^{2}(0, L)}^{2}\right\} d t \\
& \leqslant \int_{0}^{T}\left\{\left(\sup _{t \in[0, T]}\|\varphi(t)\|_{\mathrm{H}_{0}^{1}(0, L)}\right)^{2}+\left(\sup _{t \in[0, T]}\left\|\varphi_{t}(t)\right\|_{\mathrm{L}^{2}(0, L)}\right)^{2}\right\} d t \\
& \leqslant \int_{0}^{T}\left\{\|\varphi\|_{\mathrm{L}^{\infty}\left(0, T ; \mathrm{H}_{0}^{1}(0, L)\right)}^{2}+\left\|\varphi_{t}\right\|_{\mathrm{L}^{\infty}\left(0, T ; \mathrm{L}^{2}(0, L)\right)}^{2}\right\} d t \\
& \leqslant T\left\{\|\varphi\|_{\mathrm{L}^{\infty}\left(0, T ; \mathrm{H}_{0}^{1}(0, L)\right)}+\left\|\varphi_{t}\right\|_{\mathrm{L}^{\infty}\left(0, T ; \mathrm{L}^{2}(0, L)\right)}\right\}^{2} \\
& \leqslant T C^{2}\left\|\left\{\varphi_{0}, \varphi_{1}\right\}\right\|_{\mathrm{H}_{0}^{1}(0, L) \times \mathrm{L}^{2}(0, L)}^{2} \text {, }
\end{aligned}
$$

onde $C=C(L, T)>0$ é constante obtida com a aplicação do corolário 2.2.3. Combinando $(2.3 .20),(2.3 .21)$ e $(2.3 .22)$ obtemos

$$
\begin{aligned}
\frac{L}{2} \int_{0}^{T}\left|\varphi_{x}(L, t)\right|^{2} d t & \geqslant-|X|+\frac{1}{2} \int_{0}^{T} \int_{0}^{L}\left\{\left|\varphi_{x}(x, t)\right|^{2}+\left|\varphi_{t}(x, t)\right|^{2}\right\} d x d t \\
& \geqslant\left[-L+\frac{T}{2}\right] C^{2}\left\|\left\{\varphi_{0}, \varphi_{1}\right\}\right\|_{\mathrm{H}_{0}^{1}(0, L) \times \mathrm{L}^{2}(0, L)}^{2}
\end{aligned}
$$

ou ainda

$$
\left[T-\frac{L}{2}\right] C^{2}\left\|\left\{\varphi_{0}, \varphi_{1}\right\}\right\|_{\mathrm{H}_{0}^{1}(0, L) \times \mathrm{L}^{2}(0, L)}^{2} \leqslant \int_{0}^{T}\left|\varphi_{x}(L, t)\right|^{2} d t=\left\|\varphi_{x}(L, \cdot)\right\|_{\mathrm{L}^{2}(0, T)}^{2} .
$$

Logo, tomando $T_{0}>\frac{L}{2}$ o lema 2.3.2 segue. 


\section{Referências Bibliográficas}

[1] Robert Alexander Adams. Sobolev Spaces. Academic Press, New York, 1975.

[2] Haim Brezis. Analyse Fonctionelle Théorie et Applications. Masson, Paris, 1987.

[3] Jacques-Louis Lions e Eugene Magenes. Non-Homogeneous Boundary Value Problems and Applications, volume 1 e 2. Springer-Verlag, 1972.

[4] Luis Adauto Medeiros e Manuel Milla Miranda. Espaços de Sobolev. UFRJ IM, Rio de Janeiro, 2000.

[5] Jerome A. Goldstein. Semigroups of operators and abstract cauchy problems. Tulane University Departament of Mathematics, May 1970.

[6] Einar Hille and Ralph S. Phillips. Functional Analysis and Semi-Groups, volume 31. AMS, Providence, Rhode Island, 1957. Colloquium Publications.

[7] Jacques-Louis Lions. Contrôlabilité Exacte Perturbations et Stabilisation de Système Distribués, volume Tome 1. Masson, Paris, 1988.

[8] Luis Adauto Medeiros. Exact controllability for wave equations - hum. In Seminário Brasileiro de Análise, volume 37. SBA, 1993.

[9] Luis Adauto Medeiros. Tópicos em Equações Diferenciais Parciais. UFRJ IM, Rio de Janeiro, 2005.

[10] Michael Eugene Taylor. Partial Differential Equations. Texts in Applied Mathematics. Springer-Verlag, New York, 1996. 